T. Matsusaka

Nagoya Math. J.

Vol. 104 (1986), 175-211

\title{
ON POLARIZED NORMAL VARIETIES, I
}

\author{
T. MATSUSAKA ${ }^{1)}$
}

\section{Introduction}

In this paper, we deal with the first part of an application of our Riemann-Roch type inequalities (cf. [13], [23]) toward deformations of polarized normal varieties. In Chapter I, we discuss the problem of eliminating fixed components from complete linear systems defined by multiples of a given divisor. Let $U^{n}$ be a complete normal variety and $Y_{0}$ an ample Cartier divisor on $U$. The main result of the chapter is that there is a positive integer $c_{0}$, predicted by the first two leading coefficients of the polynomial $\chi\left(U, \mathcal{O}\left(m Y_{0}\right)\right)$, such that the complete linear system $\Lambda\left(r c_{0} Y_{0}\right)$ has no fixed component whenever $r$ is a positive integer (which is essentially contained in Theorem 1.1 (cf. [13], Lemma 5.2)). An easy consequence of this result is that when $n=2$, we can find another positive integer $c_{1}$, predicted by the same coefficients as above, such that $r c_{1} c_{0} Y_{0}$ is very ample on $U$ whenever $r$ is a positive integer. Even though this has been generalized to $n=3$ by J. Kollár (cf. [12]), we have included this in Section 3 since it is very simple.

Let $\Omega_{0}$ be the set of pairs $\left(U^{n}, Y_{0}\right)$ of normal projective varieties $U$ and ample Cartier divisors $Y_{0}$ on $U$ with a fixed polynomial $P_{0}(x)$ such that $P_{0}(m)=\chi\left(U, \mathcal{O}\left(m Y_{0}\right)\right)$. We let $Y=c_{0} Y_{0}$ for the sake of simplicity, where $c_{0}$ is as above. In Chapter II, we consider two problems.

(1) Find a positive integer $c_{1}$, depending only on the two leading coefficients of the polynomial $\chi(U, \mathcal{O}(m Y))$, such that a suitable vector subspace $L$ of the vector space $L\left(c_{1} Y\right)$ has the following two properties:

(i) A non-degenerate rational map $f$ of $U$ defined by $L$ is a birational transformation of $U$;

(ii) When $p$ denotes the projection map of the graph $T$ of $f$ on the image of $f, p^{-1}$ has no (set-theoretic) fibre of positive dimension.

Received January 14, 1985.

1) This work was done while the author was partially supported by N.S.F. 
(2) Find the estimation of $\ell(r Y)$ in the form

$$
|\ell(r Y)-\chi(U, \mathcal{O}(r Y))| \leq \phi(r),
$$

where $\phi(x)$ is a polynomial of degree at most $n-2$ with rational coeffcients, which depends only on the first two leading coefficients of $\chi(U$, $\mathcal{O}(m Y))$.

We first show that (1) has a solution, which is contained in Proposition 2.2. As a consequence of this result, we can construct a finite union $\mathfrak{B}$ of irreducible algebraic families of pairs $\left(V^{n}, X\right)$ of non-singular subvarieties $V$ of a projective space and semi-ample divisors $X$ of $V$ such that whenever $\left(U^{n}, Y_{0}\right) \in \Omega_{0}$, there is a member $(V, X)$ of $\mathfrak{B}$ and a birational morphism $\rho$ of $V$ on $U$ with $\rho^{*}\left(c_{0} Y_{0}\right) \sim X$ (Theorem 2.1). A solution of (2) which is a special case of a result of S. Cutkosky is reproduced in Theorem 2.3 for the sake of convenience. Then returning to the problem (1), these results are used to obtain a sharper result in which the vector subspace $L$ defines a linear subsystem $\Lambda\left(c_{1} Y\right)$ without fixed component (Proposition 2.5). An effect of this sharper result is contained in Theorem 2.2.

The last paragraph of Chapter II deals with an immediate application of our results. Let $\Omega_{0}^{\prime}$ be the subset of $\Omega_{0}$, consisting of those pairs $\left(U^{n}, Y_{0}\right)$ such that singularities of $U$ are rational singularities only. We show the existence of a positive integer $c$ such that whenever $\left(U, Y_{0}\right) \in \Omega_{0}^{\prime}, r c Y_{0}$ is very ample on $U$ and $h^{i}\left(U, \mathcal{O}\left(r c Y_{0}\right)\right)=0$ for $i>0$ for all positive integers $r$. The result has some close relation with [4].

Basically we shall follow definitions, terminology and conventions of [20] and [5]. Basic foundational results contained in these will be used freely without detailed references except in some delicate cases. The following is a partial list of notations and conventions which will be used without further explanations.

$K_{U} \quad$ : Canonical divisors on a normal variety $U$.

$\sim \quad$ : Linear equivalence of divisors.

$\Lambda(D) \quad$ : The complete linear system determined by a divisor $D$.

$\Lambda_{\text {red }} \quad$ : The reduced part of a linear system $\Lambda$ of divisors, i.e.

$\Lambda_{\text {red }}=\Lambda$ - the fixed part.

$L(D) \quad$ : The vector space of functions $g$ with $\operatorname{div}(g)+D>0$.

$\ell(D) \quad: \operatorname{dim} L(D)$.

$f\{W\}$ : The total transform of a subvariety or a closed subset $W$ by a rational transformation. 
$f^{-1}\{W\}$ : The complete inverse image of a closed subset $W$ by a rational transformation $f$.

$f[W] \quad$ : The proper transform of $W$ by a rational map $f$.

$f(W)$ : The intersection-theoretically defined transform of $W$ by $f$.

$Z \rightarrow Z^{\prime}$ ref. $k: \quad Z^{\prime}$ is a specialization of $Z$ over $k$.

The vanishing theorem for invertible sheaves defined by divisors of the form $K_{V}+X$ on a non-singular projective variety has been proved and generalized in [11], [6], [8], [19]. In order to avoid repeated references to these articles, we shall simply use the phrases such as "according to the vanishing theorem," "by the vanishing theorem," etc. When $W$ is a complete variety, non-singular in codimension 1 and $D$ a positive divisor on $W$, a vector subspace $L$ of $L(D)$ of positive dimension defines a nondegenerate rational map $f$ of $W$ into a projective space on one hand, it defines also a linear subsystem $A$ of $A(D)$. Conversely, $A$ defines the vector subspace $L^{\prime}$ of $L\left(D^{\prime}\right), D^{\prime} \sim D$, uniquely such that $A$ is a linear subsystem of $\Lambda(D)=\Lambda\left(D^{\prime}\right)$ defined by $L^{\prime} . \quad L$ and $L^{\prime}$ are isomorphic as vector spaces, and they define the same non-degenerate rational maps of $W$ into a projective space up to a projective transformation. Therefore we shall quite often refer to $f$ as a non-degenerate rational map of $W$ into a projective space defined by $A$.

We shall assume throughout the paper that the characteristic of the universal domain is 0 , even though some of the results are valid in characteristic $p$ too.

The author owes some improvements of the original manuscript to J. Kollár and S. Cutkosky to whom he wishes to express his appreciation.

\section{CHAPTER I}

\section{$\S 1$.}

Lemma 1.1. Let $U^{n}$ be a complete variety, non-singular in codimension 1 and $Z, D$ two positive $U$-divisors. Assume that $\ell(Z+D)>\ell(Z)$. Let $F$ be the fixed part of $A(Z+D)$ and $D=\sum a_{i} D_{i}$ the reduced expression for $D$. Then the set $I$ of indices $i$ such that the coefficient $b_{\imath}^{\prime}$ of $D_{i}$ in $F$ satisfies $b_{1}^{\prime}<a_{i}$ is not empty. Let $b_{i}=a_{i}-b_{i}^{\prime}$ and $D^{\prime}=\sum_{i} b_{i} D_{i}$. When $j \in I$ satisfies

$$
b_{j} / a_{\jmath} \geqq b_{i} / a_{i} \quad \text { for every } i \text { in } I,
$$


$D_{j}$ is not a fixed component of $\Lambda(T)$, where $T=a_{j} Z+b_{j} D$.

Proof. The first assertion is obvious. Let $B=b_{j} D-a_{3} D^{\prime} . \quad B$ is a positive $U$-divisor and $D_{3}$ is not a component of $B$. Define $A$ by $A=$ $Z+D^{\prime} . \quad D_{\jmath}$ is not a fixed component of $\Lambda(A)$ and $T=a_{j} A+B$. It follows that $D_{j}$ is not a fixed component of $\Lambda(T)$.

Corollary. With the same notations and assumptions of the Lemma, let $s=a_{j}+b_{j}$. Assume further that $D$ is the fixed part of $\Lambda(Z)$. Then $D_{l}$ is not a fixed component of $\Lambda(s Z)$.

Proof. Let $W$ be a member of $\Lambda(Z)_{\text {red }}$. As $a_{j} Z+b_{j} D+b_{j} W \sim s Z$ and $\Lambda(W)$ has no fixed component, $D_{\jmath}$ cannot be a fixed component of $\Lambda(s Z)$ by the Lemma above.

Lemma 1.2. Let $V^{n}$ be a non-singular projective variety, $X$ a numerically effective $V$-divisor with $X^{(n)}>0$ and $\Lambda$ a linear subsystem of $\Lambda(X)$ with the fixed part $F$. Assume that $X$ and $A$ satisfy the following conditions.

(i) $\ell(X)>0$ and there is a positive integer $c$ such that

$$
\left|\ell(r X)-\left(X^{(n)} / n !\right) r^{n}\right| \leqq c r^{n-1}
$$

for all positive integers $r$.

(ii) A general divisor $W$ of $\Lambda_{\text {red }}$ is absolutely irreducible and there are positive integers $c^{\prime}, c^{\prime \prime}$ such that when $r>c^{\prime}$,

$$
h^{0}(W, \mathcal{O}(r X . W)) \leqq(w /(n-1) !) r^{n-1}+c^{\prime \prime} r^{n-2},
$$

where $w=I\left(W, X^{(n-1)}\right)$.

(iii) $I\left(F, X^{(n-1)}\right)>0$.

Then there is a positive integer $e>c^{\prime}$, which depends only on $c, c^{\prime}, c^{\prime \prime}, X^{(n)}$ and $n$, such that whenever $m>e, \ell((m-j) X+F)>\ell((n-j) X)$ for some $j$ with $c^{\prime} \leqq m-j<m$.

Proof. Let $m$ be an integer with $m>c^{\prime}$ and assume that for all positive integers $j$, equalities $\ell((m-j) X+F)=\ell((m-j) X)$ hold whenever $c^{\prime} \leqq m-j<m$. The equality implies

$$
\ell(s X)=\operatorname{dim} \operatorname{Tr}_{W} L(s X)+\ell((s-1) X), \quad s=m-j,
$$

as is well known. When we sum these relations up, we find that

$$
\ell(m X) \leqq \sum_{c^{\prime}+1}^{m} h^{0}(W, \mathcal{O}(i X . W))+\ell\left(c^{\prime} X\right)
$$


Our assumption (ii) and a simple estimation then show that

$$
\ell(m X) \leqq(w / n !)(m+1)^{n}+\left(c^{\prime \prime} / n\right)(m+1)^{n-1}+u,
$$

where $u$ is a constant which depends only on $c^{\prime}, c^{\prime \prime}, X^{(n)}$ and $n . X^{(n)}-1 \geqq w$ by (iii) and $w \geqq 0$ (cf. [10]). When that is so, $e$ can be found from the above inequality and from (i).

COROLlary 1. Let the notations and assumptions be as in our Lemma and a the maximum of the coefficients of the components of $F$ in its reduced expression. Then there is a positive integer $v \leqq a(e+1)$ such that a certain component $F_{j}$ of $F$ is not a fixed component of $\Lambda(v X)$.

Proof. Let $u$ be a positive integer of the above Lemma with $u \leqq e$ such that $\ell(u X+F)>\ell(u X)$. By Lemma 1.1, there are two positive integers $a_{\jmath}, b_{j}$ satisfying $a \geqq a_{j} \geqq b_{j}>0$ such that a certain component $F_{j}$ of $F$ is not a fixed component of the complete linear system defined by $a_{j} u X+b_{j} F$. Then the same is true with the one defined by $a_{j} u X+$ $b_{\jmath} F+b_{j} W \sim\left(a_{j} u+b_{j}\right) X$. The Corollary follows from this at once.

Corollary 2. Let the notations and assumptions be as in our Lemma and assume further that $X$ is semi-ample. Let $F=F^{\prime}+F^{\prime \prime}$ in which every component $F_{i}$ (resp, $\left.F_{j}\right)$ of $F^{\prime}\left(\right.$ resp. $\left.F^{\prime \prime}\right)$ satisfies $I\left(F_{i}, X^{(n-1)}\right)=0$ (resp. $\left.I\left(F_{j}, X^{(n-1)}\right)>0\right)$. Let a be the maximal of the coefficients of components of $F^{\prime \prime}$ in its reduced expression. Then there is a positive integer $v \leqq a(e+1)$ such that a certain component of $F^{\prime \prime}$ is not a fixed component of $\Lambda(v X)$.

Proof. Let $u$ be as in the proof above. As $X$ is semi-ample, $L(t X)$ defines a birational morphism $f$ of $V$ on a normal projective variety for large $t$. Every component of $F^{\prime}$ is contracted by $f$. It follows that $\ell(u X)$ $=\ell\left(u X+F^{\prime}\right)$, and consequently $\ell\left(u X+F^{\prime}+F^{\prime \prime}\right)>\ell\left(u X+F^{\prime}\right)$. When we let $Z=u X+F^{\prime}, D=F^{\prime \prime}$ in Lemma 1.1, our Corollary follows in the same manner as the above corollary.

Remark. (I) When $X$ is semi-ample, the condition (i) of the Lemma is satisfied by our result (cf. [13]).

(II) When $n=2$, the upper bound in (ii) is obtained at once by using the Riemann-Roch theorem on curves.

(III) We shall prove in Section 2 the upper bound formula in (ii) in general when $X$ is semi-ample. But it can be obtained also for $n=2$ 
rather easily when we impose a mild condition on $W$. We shall include the result here as a Proposition.

Proposition 1.1. Let $V$ be a non-singular projective variety of dimension $3, X$ a numerically effective $V$-divisor with $X^{(3)}>0$ and $\Lambda$ a linear subsystem of $\Lambda(X)$. Let $L$ be the vector subspace of $\Lambda(X)$ which defines $\Lambda$ and assume that $L$ defines a non-degenerate rational map of $V$ with a 3-dimensional image. Denote by $T$ a general divisor of the minimum sum of $\Lambda_{\text {red }}$ taken $m$-times with $m \geqq 4$. Then

$$
h^{0}(T, \mathcal{O}(r X, T)) \leqq(x / 2) r^{2}+2, \quad x=I\left(T, X^{(2)}\right) .
$$

Proof. We may prove our result after applying a finite sequence of monoidal transformations with non-singular centers. Therefore we may assume that $\Lambda_{\text {red }}$ has no base point (cf. [7]). Let $W$ be a general divisor of $\Lambda_{\text {red }}$. We have $T \sim m W$ and both $W$ and $T$ are non-singular by the theorem of Bertini. $W$ and $T$ are semi-ample by our assumption on $L$. It follows that $\ell\left(K_{V}+u W\right)>0$ for some positive integer $u \leqq 4$ by the vanishing theorem. It follows that $\ell\left(K_{V}+T\right)>0$ by our choice of $m$ and that $p_{g}(T)>0$ by the adjunction formula.

Let $X^{\prime}=X . T$. Since we are interested in $\ell\left(r X^{\prime}\right)$, we may assume that $\Lambda\left(r X^{\prime}\right)_{\text {red }}$ has no base point by applying the same procedure as above. Let $Z$ be a general divisor of this linear system and $F$ the fixed part of $A(r X)$. By our assumption on $L$ and by a theorem of Bertini, $Z$ is an absolutely irreducible non-singular curve on $T$, and $p_{g}(T)>0$ as a monoidal transformation of a non-singular projective surface does not change $p_{g}(T)$. Then when $Z^{\prime} \sim Z$ such that $Z^{\prime} \cap Z$ is proper on $T, Z^{\prime} . Z$ is a special divisor on $Z$ and we have $\ell\left(Z^{\prime} . Z\right) \leqq(1 / 2) Z^{(2)}+1$ by the theorem of Clifford. This implies that

$$
\ell\left(r X^{\prime}\right)=\ell(Z) \leqq(1 / 2) Z^{(2)}+2 .
$$

Since $X^{\prime}$ and $Z$ are both numerically effective on $T$, we have

$$
\left(r X^{\prime}\right)^{(2)}=I\left(r X^{\prime}, Z+F\right) \geqq I\left(r X^{\prime}, Z\right) \geq Z^{(2)} .
$$

Our Proposition follows from this at once.

$\S 2$.

Let $V^{n}$ be a non-singular projective variety and $X$ a semi-ample $V$ divisor. Let $u$ be a positive integer such that $n+1 \leqq u \leqq 2 n+1$ and 
that $\ell\left(K_{V}+u X\right)>0$. Such $u$ exists by the vanishing theorem. Let $D$ be an arbitrary but fixed divisor contained in $\Lambda\left(K_{V}+u X\right)$. Then we have shown in Section 3, [13] that there is a positive divisor $D^{\prime}$ such that $D-D^{\prime}>0, I\left(D^{\prime}, X^{(n-1)}\right)=0$ and that $I\left(K_{V}+u X-D^{\prime}, Z, X^{(n-1)}\right)$ is nonnegative for all positive divisors $Z$ on $V$ in particular (cf. [13], Cor., Prop. 3.2). In order to find the upper bound formula in (ii), Lemma 1.2, in which the dependence of constants $c^{\prime}, c^{\prime \prime}$ on the polynomial $\chi(V, \mathcal{O}(m X))$ is made clear, we need the following generalization of the above result. ${ }^{2}$

Proposition 1.2. Let $V^{n}$ be a non-singular projective variety and the $A_{i} n-2$ semi-ample $V$-divisors such that $A_{1} \cap \cdots \cap A_{n-2}$ is proper on $V$. Assume that $X=\sum_{1}^{n-2} a_{i} A_{i}$ satisfies the following conditions:

(a) The $a_{i}$ are non-negative integers and $a_{i} \neq 0$ for some $i$;

(b) Whenever $D$ is a positive $V$-divisor, $I\left(D, X^{(n-1)}\right)=0$ implies

$$
I\left(D, X, A_{1} \cdots A_{n-2}\right)=0 \text { and } I\left(D, A_{i}, A_{1} \cdots A_{n-2}\right)=0
$$

for all $i$. Then there is a positive integer $u$ with $n+1 \leqq u \leqq 2 n+1$ such that $\Lambda\left(K_{V}+u X\right)$ is not empty. When $D$ is a divisor in it, there is a positive $V$-divisor $D^{\prime}$ such that $D-D^{\prime}>0, I\left(D^{\prime}, X^{(n-1)}\right)=0$ and that whenever $Z$ is a positive $V$-divisor, $I\left(K_{V}+u X-D^{\prime}, Z, A_{1} \cdots A_{n-1}\right) \geqq 0$.

We shall not give a proof of this here. Essentially a reproduction of discussions in Sections 2, 3 in [13] with a few obvious changes will prove our version of Proposition 3.2 there. Modifications needed for Sections 2, 3 in [13], Proposition 2.3 will be included in the Appendix.

Proposition 1.3. Let $V^{n}$ be a non-singular projective variety, $X$ a semiample $V$-divisor with $\ell(X)>1, \Lambda$ a linear subsystem of $\Lambda(X)$ of positive dimensions such that $\Lambda_{\text {red }}$ has no base point and $W$ a general divisor of $A_{\text {red }}$, which is assumed to be absolutely irreducible. Then

$$
I\left(K_{V}+W, W, X^{(n-2)}\right) \leqq 2^{n-1}(4 d n+2 d+\xi),
$$

where $d=X^{(n)}, \xi=I\left(K_{V}, X^{(n-1)}\right)$.

Proof. By our assumption, $X+W$ is semi-ample. There is a positive integer $u$ such that $\ell\left(K_{V}+u(X+W)\right)>0$ and that $n+1 \leqq u \leqq 2 n+1$

2) The following two Propositions are used to prove Proposition 1.4 which makes the dependence of the inequality of the Proposition on $c_{0}$ and $\chi\left(V, \mathcal{O}\left(m X_{0}\right)\right)$ explicit. The inequality can be proved using Theorem 2.1, without depending on the two Propositions, when $(V, X)$ is a member of the family $V$ of the above quoted theorem. Therefore Theorem 1.1 can be established for $(V, X) \in \mathscr{V}$ without using these two Propositions too. 
by the vanishing theorem. We shall apply Proposition $1.2 n-1$ times. First we let $A_{i}=X+W$ for all $i$. Then $A_{1}=X, A_{i}=X+W$ for $i>1$, in general $A_{1} \sim \cdots \sim A_{r} \sim X, A_{r+1} \sim \cdots \sim A_{n-2} \sim X+W$ and finally $A_{i}=X$ for all $i$. We first show that the conditions in (b) are satisfied when we let $X+W$ to be $X$ in the Proposition. When $Z$ is a positive $V$-divisor, $I\left(Z,(X+W)^{(n-1)}\right)=0$ if and only if $I\left(Z, X^{(r)}, W^{(s)}\right)=0$ for all non-negative integers $r$ and $s$ with $r+s=n-1$. When that is so, for all non-negative integers $v, w$ with $v+w=n-1, I\left(Z, X^{(v)},(X+W)^{(w)}\right)=0$. Thus our choices satisfy the condition (b) in all cases.

Our assumptions imply that we have

$$
I\left(K_{V}+W, W, X^{(n-2)}\right) \leqq I\left(K_{V^{\prime}}+u(X+W), X+W,(X+W)^{(n-2)}\right)=I_{1} .
$$

By Proposition 1.2, there is a positive divisor $D_{1}$ having the properties stated in the Proposition for $X+W$ and $A_{i}=X+W$ for all $i$. It follows that

$$
\begin{aligned}
I_{1} & =I\left(K_{V}+u(X+W)-D_{1}, X+W,(X+W)^{(n-2)}\right) \\
& \leqq 2 I\left(K_{V}+u(X+W), X,(X+W)^{(n-2)}\right) \\
& =2 I\left(K_{V}+u(X+W), X+W, X .(X+W)^{(n-3)}\right)=2 I_{2} .
\end{aligned}
$$

In general, let $I\left(K_{V}+u(X+W), X+W, X^{(r)} \cdot(X+W)^{(s)}\right)=I_{r+1}$, where $r$ and $s$ are non-negative integers such that $r+s=n-2, r<n-2$. There is a positive divisor $D_{r+1}$ on $V$ with the stated properties for $X+W$, $A_{i} \sim X(1 \leqq u \leqq r)$ and $A_{j} \sim X+W(r+1 \leqq j \leqq n-2)$ in the Proposition. Then

$$
\begin{aligned}
I_{r+1} & =I\left(K_{V}+u(X+W)-D_{r+1}, X+W, X^{(r)} \cdot(X+W)^{(s)}\right) \\
& \leqq 2 I\left(K_{V}+u(X+W), X, X^{(r)} \cdot(X+W)^{(s)}\right) \\
& =2 I\left(K_{V}+u(X+W), X+W, X^{(r+1)} \cdot(X+W)^{(s-1)}\right)=2 I_{r+2} .
\end{aligned}
$$

It follows by induction that $I_{1} \leqq 2^{n-1} I\left(K_{V}+u(X+W), X^{(n-1)}\right)$ and our Proposition follows from this.

Proposition 1.4. Let $V^{n}, n>1$, be a non-singular projective variety, $X_{0}$ a semi-ample $V$-divisor and $X=c_{0} X_{0}$. Assume that a vector subspace $L$ of $L(X)$ defines a rational transformation of $V$ with an $n$-dimensional image. Let $\Lambda$ be the linear subsystem of $\Lambda(X)$ defined by $L$ and $W$ a general divisor of $\Lambda_{\text {red }}$. Then $W$ is absolutely irreducible and there is a positive integer $c^{\prime}$ which depends only on $c_{0}$ and $\chi\left(V, \mathcal{O}\left(m X_{0}\right)\right)$ such that 


$$
h^{0}(W, \mathcal{O}(r X . W)) \leqq(w /(n-1) !) r^{n-1}+c^{\prime} r^{n-2}
$$

for all positive integers $r$, where $w=I\left(W, X^{(n-1)}\right)$.

Proof. $W$ is absolutely irreducible by our assumption on $L$ and by the theorem of Bertini. Let $g: V^{\prime} \rightarrow V$ be a birational morphism such that $g^{-1}$ is a succession of monoidal transformations with non-singular centers and that $L^{\prime}=\{f \circ g, f \in L\}$ defines a linear system $\Lambda^{\prime}$ on $V^{\prime}$ without base point (cf. [7]). $g^{-1}[W]=W^{\prime}$ is a general divisor of $\Lambda^{\prime}$. When we let $X^{\prime}=g^{-1}(X)$, we have $X^{(n)}=X^{\prime(n)}, I\left(K_{V}, X^{(n-1)}\right)=I\left(K_{r^{\prime}}, X^{(n-1)}\right)$ and $I\left(W, X^{(n-1)}\right)=I\left(W^{\prime}, X^{(n-1)}\right)$. It follows that there is a positive integer $s$ which depends only on $c_{0}$ and $\chi\left(V, \mathcal{O}\left(m X_{0}\right)\right)$ such that $I\left(K_{W^{\prime \prime}}, X^{\prime(n-2)}\right) \leqq s$ by Proposition 1.3. Next we shall find a lower bound for $I\left(K_{W^{\prime}}, X^{\prime(n-2)}\right)$. $W^{\prime(n)}>0$ by our assumption and $W^{\prime}$ is semi-ample on $V^{\prime}$. It follows that $u X_{0}^{\prime}+W^{\prime}$ is also semi-ample for all positive integers $u$, where $X_{0}^{\prime}=g^{-1}\left(X_{0}\right)$. We can find a positive integer $u$ such that $u \leqq n+1$ and that $\ell\left(K_{r^{\prime \prime}}, u X_{0}^{\prime}\right)$ $>0$ by the vanishing theorem. Fixing one such $u$, we find that

$$
I\left(K_{V^{\prime}}+u X_{0}^{\prime}+W^{\prime}, W^{\prime}, X^{\prime(n-2)}\right)=I\left(K_{w^{\prime}}+u X_{0}^{\prime} . W^{\prime}, X^{\prime(n-2)}\right) \geqq 0 .
$$

It follows from this that $I\left(K_{W^{\prime}}, X^{(n-2)}\right) \geqq-(n+1) c_{0}^{n-1} X_{0}^{(n)}$. Finally $0<$ $I\left(W^{\prime}, X^{\prime(n-1)}\right) \leqq X^{\prime(n)}=c_{0}^{n} X_{0}^{(n)}$. Thus we have found an upper bound $s^{\prime}$ for $I\left(W^{\prime}, X^{\prime(n-1)}\right),\left|I\left(K_{W^{\prime}}, X^{\prime(n-2)}\right)\right|$ which depends only on $c_{0}$ and the polynomial $\chi\left(V, \mathcal{O}\left(m X_{0}\right)\right)$.

When that is so, there is a finite set $\left\{P_{i}(x)\right\}$ of polynomials determined by $s^{\prime}$ such that one of them is the polynomial $\chi\left(W^{\prime}, \mathcal{O}\left(m X^{\prime}, W^{\prime}\right)\right)$ by Lemma 5.2 , [13]. Then we can find a positive integer $c^{\prime}$, depending only on $c_{0}$ and $\chi\left(V, \mathcal{O}\left(m X_{0}\right)\right)$ such that

$$
h^{0}\left(W^{\prime}, \mathcal{O}\left(r X^{\prime} \cdot W^{\prime}\right)\right) \leqq(w /(n-1) !) r^{n-1}+c^{\prime} r^{n-2}
$$

by the main theorem in [13]. As $h^{\circ}(W, \mathcal{O}(r X . W)) \leqq h^{\circ}\left(W^{\prime}, \mathcal{O}\left(r X^{\prime} . W^{\prime}\right)\right)$, our Proposition is proved.

LEMMA 1.3. Let $V^{n}$ be a non-singular projective variety and $X$ a semi-ample $V$-divisor. When a vector subspace $L$ of $L(m X)$ satisfies $\operatorname{dim} L \geqq$ $X^{(n)} m^{n-1}+n, L$ defines a non-degenerate rational map of $V$ with an $n$-dimensional image.

Proof. This is an easy consequence of [15], Theorem 3 and a similar proof as [16], Proposition 2.5 works. 
THEOREM 1.1. Let $V$ be a non-singular projective variety of dimension $n>1$ and $X_{0}$ a semi-ample $V$-divisor. Then we can find a positive integer $c^{*}$, depending only on $\chi\left(V, \mathcal{O}\left(m X_{0}\right)\right)=P_{0}(m)$, such that for some positive integer $r \leqq c^{*}$ the complete linear system $\Lambda\left(r X_{0}\right)$ has no fixed component $G$ with $I\left(G, X_{0}^{(n-1)}\right)>0$ and $L\left(r X_{0}\right)$ defines a non-degenerate rational map of $V$ with an n-dimensional image.

Proof. We can find a positive integer $\bar{c}$, depending only on the polynomial $P_{0}(x)$, such that

$$
\left|\ell\left(m X_{0}\right)-\left(X_{0}^{(n)} / n !\right) m^{n}\right| \leqq \bar{c} m^{n-1}
$$

for all positive integers $m$ (cf. [13]). Therefore we can find a positive integer $c_{0}$, depending only on $P_{0}(x)$, such that $L\left(c_{0} X_{0}\right)$ defines a rational map of $V$ with an $n$-dimensional image by Lemma 1.3. This shows that when we let $X=c_{0} X_{0}$ and $c=\bar{c} c_{0}^{n-1}$, the condition (i) of Lemma 1.2 is satisfied by $V$ and $X_{0}$.

Let $W$ be a general divisor of $\Lambda(X)_{\text {red }}$ and $F$ the fixed part of $\Lambda(X)$. Since $n>1, W$ is absolutely irreducible by a theorem of Bertini and by our choice of $c_{0}$. Moreover, Proposition 1.4 shows that a positive integer $c^{\prime}$ can be found, depending only on $P_{0}(x)$, so that the latter part of Lemma 1.2 , (ii) is also satisfied for all positive integers $r$. Therefore we may apply Lemma 1.2 when $I\left(F, X^{(n-1)}\right)>0$. The set of distinct fixed components of $\Lambda(t X)$ forms a non-increasing sequence of sets as $t$ increases. Let $G$ be the set of those components $G_{i}$ of $F$ with $I\left(G_{i}, X^{(n-1)}\right)>0$ and $a$ the maximum among the coefficients of the $G_{i}$ in the reduced expression for $F$. $\quad a$ and the number of distinct $G_{i}$ are both bounded by $I\left(X, X_{0}^{(n-1)}\right)=$ $c_{0} X_{0}^{(n)}$. We can now apply Lemma 1.2 and its Corollary 2 to our situation. According to them, there is a positive integer $c^{\prime \prime}$, which depends only on $P_{0}(x)$, such that some $G_{i}$ in $G$ is not a fixed component of $\Lambda(v X)$ for some positive integer $v \leq a\left(c^{\prime \prime}+1\right)$. Our proof will be completed when we replace $X$ by $v X$ and repeat the above process at most $c_{0} X_{0}^{(n)}$ times.

\section{§3. An application}

Let $U$ be a normal projective surface and $Y_{0}$ an ample Cartier divisor on $U$. Let $V$ be a non-singular projective surface and $g$ a birational morphism of $V$ on $U$. When we let $X_{0}=g *\left(Y_{0}\right), \chi\left(V, \mathcal{O}\left(m X_{0}\right)\right)$ is determined by the two leading coefficients $d_{0}, \xi_{0}$ of $\chi\left(U, \mathcal{O}\left(m Y_{0}\right)\right)$ up to finitely many ambiguities (cf. [13], Lemma 5.2). Therefore, we can find a positive integer 
$c$, depending only on $d_{0}, \xi_{0}$, such that $\Lambda\left(r Y_{0}\right)$ has no fixed component and $L\left(r Y_{0}\right)$ defines a rational map of $U$ with a 2-dimensional image for some positive integer $r \leq c$ by Theorem 1.1. Let $Y^{\prime} \sim r Y_{0}$ and we choose $Y^{\prime}$ so that $\operatorname{supp}\left(Y^{\prime}\right)$ does not contain a singular point of $U$. This is possible as $Y_{0}$ is a Cartier divisor on a normal projective surface $V$. Let $Y$ be a general divisor of $\Lambda\left(Y^{\prime}\right)$, which is absolutely irreducible by a theorem of Bertini.

The problem of finding a positive integer $e$, depending only on $d_{0}$ and $\xi_{0}$, such that $\Lambda(m Y)$ has no base point and that $L(m Y)$ defines a projective embedding for $m>e$ was solved by us. Subsequently, J. Kollár generalized it to dimension 3 (cf. [12]). Moreover, the case of normal surfaces can be deduced from his proofs. However his proofs are rather complex. Therefore it may be worthwhile to discuss brief outline of our original proof for normal surfaces here as it is rather simple.

First note that $p_{a}(m Y)=(1 / 2) Y^{(2)} m^{2}+(1 / 2) I\left(K_{U}, Y\right) m+1$ as $Y$ is a Cartier divisor. Then we consider an exact sequence of invertible sheaves

$$
0 \longrightarrow \mathcal{O}\left(i Y^{\prime}-Y\right) \longrightarrow \mathcal{O}\left(i Y^{\prime}\right) \longrightarrow \mathcal{O}_{Y}(i \mathfrak{y}) \longrightarrow 0,
$$

where $\mathfrak{l}=Y^{\prime} . Y$. In the cohomology exact sequence derived from the above, let $\mu_{i}$ be the homomorphism: $H^{0}(U, \mathcal{O}(i Y)) \rightarrow H^{0}(Y, \mathcal{O}(i \eta))$. We note two basic facts on curves with singularities. (a) When $\operatorname{deg}(i$ i $)>2 p_{a}(Y)$

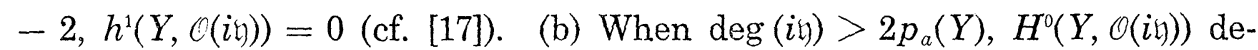
fines a complete linear system $A(i$ ) $)$ of Cartier divisors containing a positive $Y$-divisor of the same degree as in (cf. [15]). Consequently it is base point free when $\operatorname{deg}(i \xi)>2 p_{a}(Y)+1$.

We have an upper bound formula $\ell(m Y) \leqq d_{0} r^{2} m^{2} / 2+\bar{c} m$, where $\bar{c}$ is a positive integer which depends only on $d_{0}, \xi_{0}$ and $r$ (cf. [13]). Let $c_{1}$ be the smallest positive integer such that $\operatorname{deg}\left(c_{1} \mathfrak{y}\right)>I\left(K_{U}+Y, Y\right)=2 p_{a}(Y)-2$. Then an implication of (a) above is that $h^{2}(U, \mathcal{O}(j Y))=0$, that $\left\{h^{1}(U, \mathcal{O}(i Y))\right\}$ is monotone non-increasing and that $h^{1}(U, \mathcal{O}(j Y)) \leqq \bar{c} c_{1}$ for $j \geqq c_{1}$. Take $j \geqq c_{1}+2$. If $h^{1}(U, \mathcal{O}(j Y))=h^{1}(U, \mathcal{O}(j Y+Y))$ for such $j, \mu_{j+1}$ is surjective and $\Lambda((j+1) Y)$ has no base point because of $(b)$. If the above equality does not occur until $j=c_{1}+2+\bar{c} c_{1}$, the $h^{1}(U, \mathcal{O}(j Y))$ decrease strictly and $h^{1}(U, \mathcal{O}(j Y))=0$ for all $j \geqq c_{1}+2+\bar{c} c_{1}$. In this case, $\mu_{j+1}$ is surjective for all such $j$ and $\Lambda((j+1) Y)$ as no base point. It follows that there is a positive integer $s \leqq c_{1}+3+\bar{c} c_{1}$ such that $\Lambda(m s Y)$ has no base point for all positive integers $m$. Then the existence of $e$ with the stated property 
follows from [10], Chap. II, $\S 1$, Proposition 1 and Theorem 1 , since $U$ is normal.

\section{Chapter II}

\section{$\S 1$.}

First we shall review the concept of variable cycles of a linear system. Let $W^{n}$ be a variety which is non-singular in codimension 1 and $k$ a field of definition of $W$. Let $\Lambda$ be a linear system of $W$-divisors, defined over $k$, defined by a vector space $L$ of functions on $W$ in the function field over $k$. Denote by $h$ a non-degenerate rational map of $W$ into a projective space $P$ defined by $L$ and let $H_{1}, \cdots, H_{s}$ be independent generic hyperplanes in $P$ over $k$. The cycle $h^{-1}\left(H_{1} \cdots H_{s}\right)$ defined in terms of the intersection theory is by definition a variable $(n-s)$-cycle of $\Lambda$ over $k$. It is a strictly positive $W$-cycle when $s \leqq \operatorname{dim} \operatorname{Im} h$. Otherwise it is the zero cycle. Numerical equivalence class defined by $h^{-1}\left(H_{1} \cdots H_{s}\right)$ is denoted by $\Lambda^{[n-s]}$ when $W$ is non-singular. The variable cycle of $\Lambda$ over $k$ is uniquely determined, up to a generic specialization over $k$.

An alternative definition which does not use $h$ is as follows. Let the $Z_{i}$ be $s$ independent generic divisors of $A$ over $k$. Let the $D_{i}$ be the proper components of $Z_{1} \cap \cdots \cap Z_{s}$ on $W$ with the multiplicities $d_{i}$ such that the $k$-closures of the $\operatorname{supp}\left(D_{i}\right)$ are $W$. Then $\sum d_{i} D_{i}=h^{-1}\left(H_{1} \cdots H_{s}\right)$ when the $H_{i}$ are suitably chosen. We need the following elementary but basic fact concerning the variable cycles $^{3)}$.

LEMMA 2.1. Let $W^{n}$ be a projective variety, non-singular in codimension $1, A$ a linear system of $W$-divisors and $\Lambda^{\prime}$ a linear subsystem of $\Lambda$. Let $k$ be a common field of definition of $W, \Lambda$ and $\Lambda^{\prime}$; let further $J$ and $J^{\prime}$ be variable s-cycles of $\Lambda$ and $\Lambda^{\prime}$ over $k$. Then there is a positive s-cycle $R$ on $W$ such that $J^{\prime}+R$ is a specialization of $J$ over $k$.

Proof. Let $Z_{1}, \cdots, Z_{n-s}$ (resp. $Z_{1}^{\prime}, \cdots, Z_{n-s}^{\prime}$ ) be independent generic divisors of $\Lambda$ (resp. $\Lambda^{\prime}$ ) over $k$. We may assume, without loss of generality, that

3) Lemma 1.2 in [16] is valid only for $r=0$ as stated and proved there. [16] uses only this case of the Lemma. The following Lemma and the Corollaries which follow are general results valid for all $r=s$ with $0 \leqq s \leqq n$. 


$$
Z_{1} \cdots Z_{n-s}=J+\Delta, \quad Z_{1}^{\prime} \cdots Z_{n-s}^{\prime}=J^{\prime}+\Delta^{\prime}
$$

where $\Delta$ (resp. $\Delta^{\prime}$ ) is a positive $s$-cycle on $W$ such that supp $(\Delta)$ (resp. $\left.\operatorname{supp}\left(\Delta^{\prime}\right)\right)$ is contained in the base locus of $\Lambda$ (resp. $\left.\Lambda^{\prime}\right) .\left(Z^{\prime}\right)$ is a specialization of $(Z)$ over $k$. Let

$$
J+\Delta \longrightarrow A+\bar{J} \text { ref. } k
$$

be an arbitrary extension of the specialization $(Z) \rightarrow\left(Z^{\prime}\right)$ ref. $k$ to that of $J+\Delta$, where $\bar{A}$ denotes a specialization of $\Delta$ over $k$. Let $E^{\prime}$ be a proper component of $Z_{1}^{\prime} \cap \cdots \cap Z_{n-s}^{\prime}$, containing a generic point of $W$ over $k$ (i.e. the $k$-closure of $\operatorname{supp}\left(E^{\prime}\right)$ is $W$ ) of multiplicity $e$. Then $E^{\prime}$ is a component of $J^{\prime}$ of multiplicity $e$ and vice versa. It follows that $E^{\prime}$ is a component of $A$ of multiplicity $e$ (cf. [18], Theorem 11), since no component of $\bar{J}$ can contain a generic point of $W$ over $k$. This implies that $A-J^{\prime}>0$. This proves our lemma.

Corollary 1. With the same notations and assumptions of the above Lemma, let $D$ be an ample Cartier divisor on $W$. Then we have

$$
I\left(D^{(s)}, \Lambda^{[n-s]}\right) \geq I\left(D^{(s)}, \Lambda^{[n-s]}\right) .
$$

CoROLLARY 2. With the same notations and assumptions of the above Lemma, assume further that $W$ is non-singular. Let $D$ be a numerically effective divisor on $W$. Then we have $I\left(D^{(s)}, \Lambda^{[n-s]}\right) \geq I\left(D^{(s)}, \Lambda^{[n-s]}\right)$.

Proofs. These two Corollaries follow from the Lemma and from the compatibility of specializations with the operation of intersection-product (cf. [18], Theorem 11).

\section{$\S 2$.}

We shall fix the following notations and assumptions throughout the remainder of this Chapter. $\Omega_{0}$ denotes the set of pairs $\left(U, Y_{0}\right)$ of normal projective varieties of dimension $n>1$ and ample Cartier divisors $Y_{0}$ on $U$ such that the polynomial $P_{0}(x)$ defined by $P_{0}(m)=\chi\left(U, \mathcal{O}\left(m Y_{0}\right)\right)$ is shared by all members of $\Omega_{0}$. Let $d_{0}=Y_{0}^{(n)}, \xi_{0}=I\left(K_{U}, Y_{0}^{(n-1)}\right)$. When $\rho$ is a birational morphism of a projective non-singular variety $V$ on $U$ and $\rho^{*}\left(Y_{0}\right)$ $=X_{0}$, we have $d_{0}=X_{0}^{(n)}, \xi_{0}=I\left(K_{V}, X_{0}^{(n-1)}\right)$ and $\chi\left(V, \mathcal{O}\left(m X_{0}\right)\right)$ is uniquely determined by $d_{0}$ and $\xi_{0}$ within finitely many ambiguities (cf. [13], Lemma 5.2). And there is a polynomial $\phi_{0}(x)$ with rational coefficients of degree at most $n-1$, which depends only on $d_{0}$, $\xi_{0}$, such that 


$$
\left|\ell\left(r Y_{0}\right)-d_{0} r^{n} / n !\right| \leq \phi_{0}(r)
$$

for all positive integers by [13]. By [16] and its generalization in [14], there is a positive integer $c_{0}^{\prime}$ which depends only on $d_{0}$, $\xi_{0}$ such that $L\left(m c_{0}^{\prime} Y_{0}\right)$ defines a birational transformation of $U$ for all $m>0$. When we apply Theorem 1.1 to our situation, a positive integer $c_{0}$, which is a multiple of $c_{0}^{\prime}$, can be determined depending only on $P(x)$ such that $\Lambda\left(m c_{0} Y_{0}\right)$ contains an absolutely irreducible divisor ${ }^{4}$. We let $Y=c_{0} Y_{0}$ and denote by $\Omega$ the set of pairs $(U, Y)$ with $\left(U, Y_{0}\right) \in \Omega_{0}$. We define $\phi(x)$ by $\phi(x)=$ $\phi_{0}\left(c_{0} x\right)$ and $P(x)$ by $P(x)=P_{0}\left(c_{0} x\right)$. The first two leading coefficients of $P(x)$ are $d=c_{0}^{n} d_{0}, \xi=c_{0}^{n-1} \xi_{0}$ and inequalities above for $(U, Y)$ of $\Omega$ can be expressed as

$$
\left|\ell(r Y)-d r^{n} / n !\right| \leq \phi(r) .
$$

From now on, we shall fix one $(U, X) \in \Omega$ until the end of this section.

We fix the smallest positive integer $c_{1}$ such that

$$
d m^{n} / n !-\phi(m)-\sum_{1}^{n-1} Y^{(n)} m^{j}>Y^{(n)} m^{n-1}+n>0
$$

for all positive integers $m \geqq c_{1}$. $\quad c_{1}$ depends only on $d$ and $\xi$.

The main purpose of this section is to look for a vector subspace $L$ of $L(m Y)$, for some $m \geqq c_{1}$, which defines a birational transformation of $U$ satisfying the condition (c) of Proposition 2.1. For this purpose, we start with a vector subspace $L_{0}$ (resp. linear subsystem $\Lambda$ defined by $L_{0}$ ) of $L(m Y)$ (resp. $\Lambda(m Y)$ ) satisfying

$$
\operatorname{dim} L_{0}>Y^{(n)} m^{n-1}+n \quad\left(\text { resp. } \operatorname{dim} \Lambda>Y^{(n)} m^{n-1}+n-1\right) .
$$

Let $f_{0}$ be a non-degenerate rational map of $U$ into a projective space $P^{\prime}$ defined by $L_{0}$. We shall make the following assumption.

Assumption (A). The graph $T$ of $f_{0}$ contains a subvariety $F^{n-r}$ such that its projection $F^{\prime}$ in $P^{\prime}$ has dimension $n-r-j, j>0$.

Let $k$ be a common field of definition for $U, T$ and $W$ the image of $f_{0}$. The above assumption is equivalent to the following:

There is a point $w^{\prime}$ in $W$ such that $\operatorname{dim}\left(T \cap U \times w^{\prime}\right)>0$. It is also equivalent to the following:

There is a point $\left(u^{\prime} w^{\prime}\right)$ on $T$ such that $\operatorname{dim}_{k} k\left(u^{\prime}, w^{\prime}\right)>\operatorname{dim}_{k} k\left(w^{\prime}\right)$.

4) The results of $\S 2, \S 3$ will not use the fact that $\Lambda\left(m c_{0} Y_{0}\right)$ contains an absolutely irreducible divisor. cf. Footnote 2). 
Among subvarieties of $T$ which satisfy the condition in (A), there is a maximal one. Among such maximal subvarieties, we choose one with the maximum dimension. One such subvariety will be denoted by $F$. As above we let $\operatorname{dim} F=n-r, F^{\prime}$ the projection of $F$ in $P^{\prime}$ and $\operatorname{dim} F^{\prime}=$ $n-r-j$.

LEMMA 2.2. Let $k$ be a common field of definition for $U, f_{0}, F$ over which $Y$ is rational and $y$ a generic point of $F^{\prime}$ over $k$. Let $p_{2}$ be the projection of $T$ into $P^{\prime}$. Then $\operatorname{dim} p_{2}^{-1}\{y\}=j$. Moreover there is a component $A$ of $p_{2}^{-1}\{y\}$ contained in $F$, and $\operatorname{dim} A=j$.

Proof. Assume that there is a component $B$ of $p_{2}^{-1}\{y\}$ with $\operatorname{dim} B>j$. When $G$ denotes the $k$-closure of $\operatorname{supp}(B)$, we have $\operatorname{dim} G=\operatorname{dim} B+$ $\operatorname{dim} F^{\prime}>n-r$ and its projection in $P^{\prime}$ is $F^{\prime}$. This is against the choice of $F$. Let $p_{2}^{\prime}$ be the projection of $F$ into $P^{\prime}$. Every component $A$ of $p_{2}^{\prime-1}\{y\}$ has dimension $j$ and is contained in $p_{2}^{-1}\{y\}$. It follows that $A$ is also a component of $p_{2}^{-1}\{y\}$. This proves the Lemma.

LEMMA 2.3. Using the same notations and assumption of the above Lemma, let the $H_{i}$ be $n-j$ independent generic hyperplanes in $P^{\prime}$ over $k(y)$, containing $y$. When $P$ denotes the ambient projective space of $U$,

$$
T \cap P \times H_{1} \cap \cdots \cap P \times H_{i}, \quad 1 \leqq i \leqq n-j
$$

is a proper intersection in $P \times P^{\prime}$. When $A$ is a component of $P_{2}^{-1}\{y\}$ with $\operatorname{dim} A=j$, it is a component of the above intersection for $i=n-j$.

Proof. $T \cap P \times H_{1}$ is clearly proper on $P \times P^{\prime}$ as $\operatorname{dim} \operatorname{Im} f=n$ by Lemma 1.3 and by (2). Assume that the above intersection is proper for $i \leqq s<n-j$ and let $W_{s}$ be the intersection for $i=s$. $W_{s} \cap P \times H_{s+1}$ is not proper in $P \times P^{\prime}$ if and only if a component of $W_{s}$ has the projection which is contained in $H_{s+1}$. By the definition of the $H_{t}$, this is possible if and only if a component $B$ of $W_{s}$ has the projection $y$ in $P^{\prime}$. As $\operatorname{dim} B \leqq j$ by Lemma 2.2 , this is impossible when we count the dimension of the intersection. $A$ is contained in the intersection for all $i$. Since the intersection is proper in $P \times P^{\prime}$ for $i=n-j$ as we have shown above, the last assertion is also proved.

LEMMA 2.4. Under the assumption (A), we can find a vector subspace $L_{1}$ of $L_{0}$ which defines a linear subsystem $\Lambda_{1}$ of $\Lambda$, having the following properties: 
(a) $\operatorname{dim} L_{1}=\operatorname{dim} L_{0}-1$.

(b) There is a positive integer $j$ with $j<n$, and a common field $k^{\prime}$ of definition for $U, A, \Lambda_{1}$ such that when $J, J_{1}$ respectively denote variable $j$ cycles of $A, \Lambda_{1}$ over $k^{\prime}$, there is a strictly positive cycle $\Delta$ in the ambient projective space $P$ of $U$ such that $J_{1}+\Delta$ is a specialization of $J$ over $k^{\prime}$.

Proof. We shall use the same notations and assumptions of the above two Lemmas. The subset $\Lambda_{1}^{\prime}$ of $\Lambda_{\text {red }}$, consisting of $\operatorname{pr}_{U}[T .(U \times H)]$ for all hyperplanes $H$ in $P^{\prime}$ containing $y^{\prime}$, defines a linear subsystem of $A_{\text {red }}$ of dimension 1 less. We claim that $\Lambda_{1}=\Lambda_{1}^{\prime}+$ (the fixed part of 1 ) has the properties (a), (b) of the Lemma.

Let the $H_{j}^{*}$ be $n-j$ independent generic hyperplanes in $P^{\prime}$ over $k^{\prime}(y)$. $\operatorname{pr}_{U}\left[T .\left(U \times H_{1}^{*} \cdots H_{n-j}^{*}\right)\right]$ is a variable $j$-cycle of $\Lambda$ over $k^{\prime}(y)$. It is absolutely irreducible since $\operatorname{dim} \operatorname{Im} f_{0}=n$ by Lemma 1.3 and $j>0$. Moreover

$$
\operatorname{pr}_{U}\left[T .\left(U \times H_{1}^{*} \cdots H_{n-j}^{*}\right)\right]=\operatorname{pr}_{P}\left[T .\left(P \times H_{1}^{*} \cdots H_{n-j}^{*}\right)\right]
$$

as the latter is also absolutely irreducible as $\operatorname{dim} \operatorname{Im} f_{0}=n$ and $j>0$ by a theorem of Bertini. Hence the latter is a variable $j$-cycle of $A$ over $k^{\prime}(y)$.

Next we show that

$$
\operatorname{pr}_{P}\left[T .\left(P \times H_{1} \cdots H_{n-j}\right)\right] \geqq J_{1}+\operatorname{pr}_{P} A .
$$

$T$ and $P \times H_{1} \cdots H_{n-j}$ intersect properly on $P \times P^{\prime}$ and a component $A$ of the intersection is a component of $p_{2}^{-1}\{y\}$, contained in $F$ by Lemmas 2.1, 2.2. As $A$ projects to the point $y$ in $P^{\prime}, \operatorname{pr}_{P} A$ is a strictly positive cycle in $P$ and is a component of the left hand side above. Letting $Z_{i}=\operatorname{pr}_{U}\left[T .\left(U \times H_{i}\right)\right]$, we compare three cycles $\operatorname{pr}_{P}\left[T .\left(P \times H_{1} \cdots H_{n-j}\right)\right]$, $\operatorname{pr}_{U}\left[T .\left(U \times H_{1} \cdots H_{n-j}\right)\right]$ and $Z_{1} \cdots Z_{n-j}$. First we have an obvious relation

$$
\operatorname{pr}_{P}\left[T .\left(P \times H_{1} \cdots H_{n-j}\right)\right] \geqq \operatorname{pr}_{U}\left[T .\left(U \times H_{1} \cdots H_{n-j}\right)\right],
$$

when the latter is identified with a $P$-cycle. Also we have

$$
\operatorname{pr}_{U}\left[T .\left(U \times H_{1} \cdots H_{n-j}\right)\right]=Z_{1} \cdots Z_{n-j}+E,
$$

where every component of $E$ has the property that $f_{0}$ is not defined along it (cf. [20], Chapter VIII, Theorem 11 and Lemma 2.2). Finally, as the $Z_{i}$ are independent generic divisors of $\Lambda_{1 \text {, red }}$, over $k^{\prime}(y)$, we have

$$
Z_{1} \cdots Z_{n-\jmath} \geqq J_{1}
$$

where $J_{1}$ denotes a variable $j$-cycle of $\Lambda_{1}$ over $k^{\prime}(y)$. Therefore every com- 
ponent of $J_{1}$ contains a generic point of $U$ over $k^{\prime}(y)$. Then these three facts imply that $\operatorname{pr}_{P}\left[T .\left(P \times H_{1} \cdots H_{n-j}\right)\right]-J_{1} \geqq 0$. As $\mathrm{pr}_{P} A$ is algebraic over $k^{\prime}(y)$, our assertion has been proved.

$H_{1} \cdots H_{n-j}$ is a specialization of $H_{2}^{*} \cdots H_{n-j}^{*}$ over $k^{\prime}\left(y^{\prime}\right)$. Then

$$
\operatorname{pr}_{P}\left[T .\left(P \times H_{1}^{*} \cdots H_{n-\jmath}^{*}\right)\right] \longrightarrow \operatorname{pr}_{P}\left[T .\left(P \times H_{1} \cdots H_{n-j}\right)\right] \text { ref. } k^{\prime}(y)
$$

by the compatibility of specializations with the operations of intersectionproduct and algebraic projection (cf. [18]). Since the former is a variable $j$-cycle $J$ of $A$ over $k^{\prime}(y)$, our Lemma has been proved.

Corollary.

$$
\begin{aligned}
I\left(J_{1}, Y^{(j)}\right) & \leqq I\left(J, Y^{(j)}\right)-1 \\
I\left(\Lambda_{1}^{[t]}, Y^{(t)}\right) & \leqq I\left(\Lambda^{[t]}, Y^{(t)}\right) \leqq Y^{(n)} m^{n-t}
\end{aligned}
$$

in general.

Proof. The first inequality follows from our Lemma and from the compatibility of specializations with the operation of intersection-product (cf. [18]). The second inequalities follow from Lemma 2.1 and from the fact that $A$ is a linear subsystem of $\Lambda(m Y)$.

Proposition 2.1. Let $m$ be an arbitrary positive integer satisfying $m \geqq c_{1}$. Then there is a positive integer $s$ with the following properties:

(a) $s \geqq \sum_{1}^{n-1} Y^{(n)} m^{i}$.

(b) There is a linear subsystem $\Lambda^{*}$ of $\Lambda(m Y)$ such that

$$
\operatorname{dim} \Lambda^{*}=\operatorname{dim} \Lambda(m Y)-s
$$

and that $\Lambda^{*}$ defines a rational map $\tilde{f}$ of $U$ into a projective space such that $\operatorname{dim} \operatorname{Im} \tilde{f}=n$.

(c) Let $\operatorname{Im} \tilde{f}=U^{\prime}$ and $T$ the graph of $\tilde{f}$ on $U \times U^{\prime}$. Then every point $u^{\prime}$ of $U^{\prime}$ satisfies $\operatorname{dim}\left(T \cap U \times u^{\prime}\right)=0$.

Proof. Assume that a non-degenerate rational map $\bar{f}$ defined by $\Lambda(m Y)$ satisfies the assumption (A). Otherwise, there is nothing to prove. Then there is a linear subsystem $\Lambda_{1}$ of $\Lambda(m Y)$, having the properties described in Lemma 2.4 and its Corollary. By our choice of $m$ (cf. (1)), we have

$$
\begin{gathered}
\operatorname{dim} \Lambda_{1}=\operatorname{dim} \Lambda(m Y)-1 \geqq Y^{(n)} m^{n-1}+n-1>0, \\
\sum_{1}^{n-1} I\left(A_{i}^{[i]}, Y^{(i)}\right) \leqq \sum_{1}^{n-1} Y^{(n)} m^{i}-1 .
\end{gathered}
$$


Assume that a linear subsystem $\Lambda_{t}$ of $\Lambda_{t-1}$ has been already defined by applying Lemma 2.4 , due to the fact that the $\Lambda_{i}$, for $i<t$, satisfy the assumption (A). Then $\Lambda_{t}$ satisfies

$$
\begin{gathered}
\sum_{1}^{n-1} I\left(\Lambda_{t}^{[i]}, Y^{(i)}\right) \leqq \sum_{1}^{n-1} Y^{(n)} m^{i}-t, \\
\operatorname{dim} \Lambda_{t}=\operatorname{dim} \Lambda(m Y)-t .
\end{gathered}
$$

Since the hand side of the first inequality is non-negative, $t$ satisfies

$$
t \leqq \sum_{1}^{n-1} Y^{(n)} m^{i}
$$

When that is so, (1) and Lemma 1.3 show that a non-dengerate rational map $f_{t}$ of $U$ defined by $\Lambda_{t}$ has the property that $\operatorname{dim} \operatorname{Im} f_{t}=n$.

Assume further that the assumption (A) is still valid for $f_{t}$. Then $\Lambda_{t+1}$ can be found by applying Lemma 2.4. $\Lambda_{t+1}$ satisfies

$$
\begin{aligned}
& \operatorname{dim} \Lambda_{t+1}=\operatorname{dim} \Lambda(m Y)-(t+1), \\
& \sum_{1}^{n-1} I\left(\Lambda_{t+1}^{[i]}, Y^{(i)}\right) \leqq \sum_{1}^{n-1} Y^{(n)} m^{i}-(t+1) .
\end{aligned}
$$

The second inequality shows that $t+1 \leqq \sum_{1}^{n-1} Y^{(n)} m^{i}$. It also shows that $\Lambda_{t+1}$ defines a non-degenerate rational map of $U$ with an $n$-dimensional image by the same reason as above and that our operations cannot be continued more than $\sum_{1}^{n-1} Y^{(n)} m^{i}$ times. It follows that there is a $\Lambda_{s}$, $s \leqq \sum_{1}^{n-1} Y^{[n]} m^{i}$ such that $\Lambda_{s}$ does not satisfy the assumption (A) and that $\Lambda_{s}$ defines a non-degenerate rational map with an $n$-dimensional image. Our Proposition is thereby proved.

In order to strengthen our result, we first prove the following lemma.

Lemma 2.5. Let $\Lambda^{*}$ and $\Lambda(m Y)$ be as in the above Proposition, $Z$ a member of $\Lambda^{*}$ and $M$ a vector subspace of $L(Z)$ which defines $\Lambda^{*}$. Let $1=g_{0}, g_{1}, \cdots, g_{r}$ be a basis of $M$ and $N$ a finite dimensional vector space of rational functions on $U$ containing 1 . Then the vector space

$$
L=\sum_{0}^{r} N \cdot g_{i}
$$

defines a non-degenerate rational map $\mu$ of $U$ with an $n$-dimensional image, and the graph of $\mu$ satisfies (c) of the Proposition. Furthermore, when $N$ defines a birational transformation of $U, \mu$ is also a birational transformation. 
Proof. The first and the last assertions are obviously true. Let $k^{\prime \prime}$ be a field of definition for $U$ satisfying the following conditions: $Y$ is rational over $k^{\prime \prime}$; the functions $g_{i}$ are in the function field $k^{\prime \prime}(U) ; N$ has a basis $1=h_{0}, h_{1}, \cdots, h_{s}$ in $k^{\prime \prime}(U)$. Then $L$ is spanned by the functions $h_{i} g_{j}$. Let $T$ be the graph of a rational map of $U$ defined over $k^{\prime \prime}$ by

$$
u \longrightarrow\left(h_{0}(u) g_{0}(u): \cdots: h_{i}(u) g_{j}(u): \cdots: h_{s}(u) g_{i}(u)\right) \text {. }
$$

In order to prove the second assertion of the Lemma, it is enough to prove that $T$ has the property (c) of the last Proposition. Let $(u, w)$ be a generic point of $T$ over $k^{\prime \prime}$ and $\left(u^{\prime}, w^{\prime}\right)$ an arbitrary point of $T$. There is a place $\zeta$ of $k^{\prime \prime}(u, w)$ over $k^{\prime \prime}$ such that $\zeta(u, w)=\left(u^{\prime}, w^{\prime}\right)$. There are indices $p, q$ such that $\zeta$ is finite on

$$
\left(\cdots, h_{i}(u) / h_{p}(u), \cdots\right), \quad\left(\cdots, g_{j}(u) / g_{q}(u), \cdots\right) .
$$

Let their images by $\zeta$ be respectively $\left(t^{\prime}\right),\left(v^{\prime}\right)$. Then $\left(\cdots, t_{i}^{\prime} v_{\jmath}^{\prime}, \cdots\right)$ is a set of affine coordinates of $w^{\prime}$ and $\left(t^{\prime}\right),\left(v^{\prime}\right)$ are contained in it as subsets. A non-degenerate rational map of $U$ defined by $M$ satisfies (c) of the last Proposition. It follows that $\operatorname{dim}_{k^{\prime \prime}} k^{\prime \prime}\left(u^{\prime},\left(v^{\prime}\right)\right) \leqq \operatorname{dim}_{k^{\prime \prime}} k^{\prime \prime}\left(\left(v^{\prime}\right)\right)$. It follows that $\operatorname{dim}_{k^{\prime \prime}} k^{\prime \prime}\left(u, w^{\prime}\right) \leqq \operatorname{dim}_{k^{\prime \prime}} k^{\prime \prime}\left(w^{\prime}\right)$. Our Lemma follows from this.

Proposition 2.2. Let $m$ be an arbitrary positive integer such that $m \geqq c_{1}$. Then there is a vector subspace $L$ of $L(m Y)$ with the following properties:

(a) A non-degenerate rational map $f$ defined by $L$ is a birational transformation;

(b) The graph of $f$ satisfies (c), Proposition 2.1.

Proof. By definition, the vector space $L(Y)$ defines a birational transformation of $U$. Let $Z, M$ and the $g_{i}$ be as in the above Lemma. When $L$ is defined by $L=\sum_{0}^{r} L(Y) g_{i}$, Lemma 2.5 above shows that $L$ has the properties (a) and (b) of our Proposition.

\section{$\S 3$.}

The main purpose of this section is to show the existence of an algebraic family $\mathfrak{B}$ of finite type such that whenever $(U, Y)$ is a member of 2 , there is a member $V$ of $\mathfrak{B}$, which is non-singular and projective, and a birational morphism $\rho$ of $V$ on $U$. In order to carry this out, we shall fix the following convention. By an irreducible algebraic family, we 
understand a triple $[W ; Z, A]$ where $W$ and $A$ are absolutely irreducible non-singular varieties, $Z$ a cycle on $W \times A$ and $Z$ has the property that $w \times A \cap Z$ is a proper intersection on $W \times A$ for every point $w$ on $W$. The members of the family consist of $Z(w)$ defined by $(w \times A) . Z=$ $w \times Z(w)$. When $[W ; Z, A]$ and $[W ; D, A]$ are two irreducible algebraic families such that $Z(w)$ is absolutely irreducible and that $D(w)$ is a cycle on $Z(w)$, we shall say that $[W ;(Z, D), A]$ is an irreducible family of pairs $(Z(W), D(w))$, etc.

Lemma 2.6. Let $(U, Y) \in \Omega, m$ an arbitrary positive integer and $f a$ non-degenerate rational map of $U$ into a projective space $P$ defined by $L(m Y)$. Then

$$
\operatorname{dim} P \leq d m^{n} / n !+\phi(m), \quad \operatorname{deg} \operatorname{Im} f \leqq Y^{(n)} m^{n} .
$$

Proof. The first inequality follows from the upper bound formula in Section 2. The second follows from the fact that the degree of the intersection-product of $n$ general divisors in $\Lambda(t m Y)$ for large $t$ is at least equal to that of the intersection-product of $n$ general hypersurface sections of $\operatorname{Im} f$ by hypersurfaces of degree $t$.

The following Lemma is well-known (cf. [16], Appendix, Lemma 8).

LEMma 2.7. Let $\mathfrak{A}$ be an irreducible algebraic family of absolutely irreducible subvarieties of a projective space $P$. Then there are two finite union of irreducible families $\cup_{i}\left[W_{i} ; B_{i}, P^{\prime}\right], \cup_{i}\left[W_{i} ; T_{i}, P \times P^{\prime}\right]$, where $P^{\prime}$ denotes a projective space, with the following properties:

(a) Whenever $w_{2}$ is a point of $W_{i}, B_{i}\left(w_{i}\right)$ is an absolutely irreducible subvariety of $P^{\prime}$, non-singular in codimension 1 , and $T_{i}\left(w_{i}\right)$ is the graph of a birational morphism of $B_{i}\left(w_{i}\right)$ on a certain member $A$ of $\mathfrak{A}$.

(b) There is a positive integers $s_{i}$ such that whenever $\left(B_{i}\left(w_{i}\right), T_{i}\left(w_{i}\right), A\right)$ is as in (a), $h$ the birational morphism of $B_{2}\left(w_{i}\right)$ on $A$ defined by $T_{i}\left(w_{i}\right)$ and whenever $C$ is a hyperplane section of $A$,

$$
s_{i} h^{*}(C) \sim L
$$

holds, where $L$ is a hyperplane section of $B_{i}\left(w_{i}\right)$.

(c) Conversely, when $A$ is a member of $\mathfrak{A}$, there is an index $i$ and a point $w_{i}$ on $W_{i}$ such that $\left(B_{i}\left(w_{i}\right), T_{i}\left(w_{i}\right), A\right)$ has the properties described in (a) and (b).

Lemma 2.8. Let $\mathfrak{A}$ be an irreducible algebraic family of absolutely irreducible subvarieties of a projective space $P$ which are assumed to be 
non-singular in codimension 1 . Then there are two finite unions of irreducible families $\cup_{i}\left[W_{i} ; B_{i}, P^{\prime}\right], \cup_{i}\left[W_{i} ; T_{i}, P \times P^{\prime}\right]$ with the following properties:

(a) Whenever $w_{i}$ is a point of $W_{i}, B_{i}\left(w_{i}\right)$ is an absolutely irreducible non-singular subvariety of $P^{\prime}$ and $T_{i}\left(w_{i}\right)$ is the graph of a birational morphism of $B_{i}\left(w_{i}\right)$ on a certain member $A$ of $\mathfrak{X}$.

(b) Let $B_{i}\left(w_{i}\right), T_{i}\left(w_{i}\right), A$ be as above and $h$ the birational morphism of $B_{i}\left(w_{i}\right)$ on $A$ defined by $T_{i}\left(w_{i}\right)$. Then there is a positive integer $t_{i}$, which depends only on the index $i$ such that $h^{-1}$ is defined by a linear subsystem of the linear system of hypersurface sections of $A$ by hypersurfaces of degree $t_{i}$, containing absolutely irreducible A-divisors.

(c) Conversely, when $A$ is a member of $\mathfrak{A}$, there is an index $i$ and a point $w_{i}$ on $W_{i}$ such that $\left(B_{i}\left(w_{i}\right), T_{i}\left(w_{i}\right), A\right)$ has the properties described in (a) and (b).

Proof. This is also well-known and we shall not go into details of a proof. It can be proved, as in the case of Lemma 2.7, using results contained in [16], Appendix, by using two facts (i) and (ii) below which are well-known too.

(i) When $[W ; T, P]$ is an irreducible family of irreducible subvarieties of $P$, the set of points $w$ on $W$ such that $T(w)$ has a singular point is a closed subset of $W$.

(ii) Let $Z$ be an absolutely irreducible subvariety of a projective space and $\Lambda(t)$ the linear system of hypersurface sections of $Z$ by hypersurfaces of degree $t$. Then a monoidal transformation of $Z$ with an absolutely irreducible non-singular center is defined by a linear subsystem of $\Lambda(t)$, containing an absolutely irreducible hypersurface section by a hypersurface of degree $t$, by taking large $t$ (cf. [21]). Moreover, finite succession of such transformations desingularize $Z$ (cf. [7]).

Theorem 2.1. There is a positive integer $c_{2}$ and a finite union $\mathfrak{B}$ of irreducible algebraic families with the following properties.

(a) Member of $\mathfrak{B}$ are of the form $(V, X)$, where $V$ is an absolutely irreducible non-singular subvariety of a fixed projective space and $X$ a positive $V$-divisor, satisfying $\operatorname{deg} X \leqq c_{2} Y^{(n)}$.

(b) When $(U, Y) \in \Omega$, there is a member $(V, X) \in \mathfrak{B}$ with a birational morphism $\rho$ of $V$ on $U$ such that $\rho^{*}(Y) \sim X$.

Proof. Using the results of Proposition 2.2, let $f$ be a non-degenerate 
birational transformation of $U,(U, Y) \in \Omega$, into a projective space which is defined by a vector subspace $L$ of $L\left(c_{1} Y\right)$, having the property (c) of Proposition 2.1. By Lemma 2.6, we may assume that $\operatorname{Im} f$ is contained in the projective space of dimension $d c_{1}^{n} / n !+\phi\left(c_{1}\right)$ with the degree $\leqq c_{1}^{n} Y^{(n)}$. Then $\operatorname{Im} f$ is contained in a finite union $\mathfrak{A}$ of irreducible algebraic families of absolutely irreducible varieties (cf. [3]).

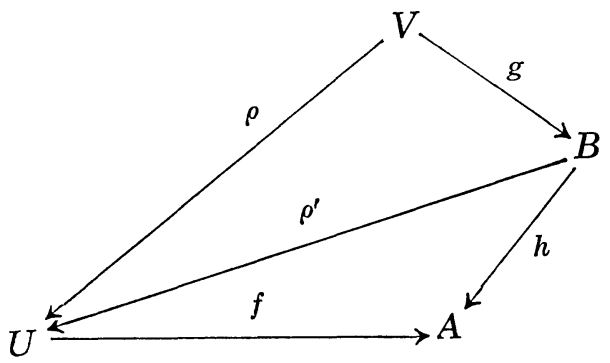

Fixing one member $(U, Y) \in \Omega$, let $f$ be as above and $A$ the image of $f$. $A$ is a member of $\mathfrak{A}$. By Lemma 2.7, there is a finite union $\mathfrak{B}$ of irreducible algebraic families corresponding to $\mathfrak{A}$ having the properties described in the Lemma. In particular, there is a member $B$ of $\mathfrak{B}$, which is nonsingular in codimension 1 and a birational morphism $h$ of $B$ on $A$. By Lemma 2.8, there is a finite union $\mathfrak{B}$ of irreducible algebraic families of non-singular subvarieties of a projective space, corresponding to the family $\mathfrak{B}$ with the properties described in the Lemma. In particular, there is a member $V$ and a birational morphism $g$ of $V$ on $B$.

Let $\rho^{\prime}=f^{-1} \circ h$ and $\rho=\rho^{\prime} \circ g$. Since $f$ has the property (c) of Proposition 2.1, $\rho$ is easily seen to be a birational morphism. Let $Y^{\prime}$ be a member of $\Lambda(Y)$ and $X=\rho^{*}\left(Y^{\prime}\right) . \quad X$ is a positive semi-ample $V$-divisor. Let $Z$ be a general divisor of the linear system without fixed component, defined by the vector space $L$. By the definition of $f$, we have $f[Z]=C_{A}$, a general hyperplane section of $A$. By Lemma 2.7, (b), there is a positive integer $s$ such that $s^{-1}\left[C_{A}\right] \sim C_{B}$ where $C_{B}$ denotes a general hyperplane section of $B$. $g^{-1}\left[C_{B}\right]=g^{-1}\left(C_{B}\right)=g^{*}\left(C_{B}\right) . \quad h^{-1}\left[C_{A}\right]$ is a general divisor of a linear system of Cartier divisors on $B$ without base point. It follows that $g^{-1}\left[h^{-1}\left[C_{A}\right]\right]=g^{*}\left(h^{-1}\left[C_{A}\right]\right)$ and that $s g^{*}\left(h^{-1}\left[C_{A}\right]\right) \sim g^{*}\left(C_{B}\right)$. By Lemma 2.8 , (b), there is a positive integer $t$ such that $\operatorname{tg}^{*}\left(C_{B}\right) \sim C_{V}+G$, where $G>0$ and $C_{V}$ is a hyperplane section of $V$. This shows that $\rho^{-1}[s t Z] \sim$ $C_{V}+G$ and hence

$$
\rho^{*}\left(\operatorname{stc}_{1} Y\right) \sim C_{V}+G+F, \quad F>0 .
$$


Let $G+F=F^{\prime}$. Note that $X$ is semi-ample on $V$ and $\rho^{*}\left(\operatorname{stc}_{1} Y\right) \sim$ stc $_{1} X$. Then, letting $C=C_{V}$, we can estimate $I\left(X, C^{(n-1)}\right)$ easily as follows:

$$
\begin{aligned}
I\left(X, C^{(n-1)}\right) & \leqq I\left(X, C+F^{\prime}, C^{(n-2)}\right)=\left(s t c_{1}\right) I\left(X^{(2)}, C^{(n-2)}\right) \\
& \leqq \cdots \leqq\left(s t c_{1}\right)^{n-1} X^{(n)}=\left(s t c_{1}\right)^{n-1} Y^{(n)}
\end{aligned}
$$

Since there are only finitely many possibilities for $s$ and $t$ by Lemma 2.7, (b) and Lemma 2.8 , (b), we define $c_{2}$ to be the maximum of $\left(s t c_{1}\right)^{n-1}$. Since $X$ is a positive divisor on $V$ with $\operatorname{deg} X \leqq c_{2} Y^{(n)}$, we can find a finite union $\mathfrak{B}^{\prime}$ of irreducible algebraic families of pairs $(V, X)$, where $V \in \mathfrak{B}$ and $X$ a positive $V$-divisor with $0<\operatorname{deg} X \leqq c_{2} Y^{(n)}$, by the main results on Chow forms and incidence correspondences (cf. [3]). This proves our Theorem.

Theorem 2.2. Assume that there is a positive integer $c_{1}^{\prime} \geqq c_{1}$, which depends only on $\Omega$, such that whenever $(U, Y) \in \Omega$, a vector subspace $L^{\prime}$ of $L\left(c_{1}^{\prime} Y\right)$ has the properties (a), (b) of Proposition 2.2 and defines a linear subsystem of $\Lambda\left(c_{1}^{\prime} Y\right)$ without fixed component. Then Theorem 2.1 is valid as it is (with possibly different $c_{2}$ and $\left.\mathfrak{B}\right)$. Moreover, there is a positive integer $c_{3}$, which depends only on $\Omega$, such that when $(V, X) \in \mathfrak{B}$ corresponds to $(U, Y) \in \Omega$ in the sense of (b), Theorem 2.1, $c_{3} X \sim C+G$, where $C$ is a very ample divisor on $V$ and every component of $G$ is contracted by $\rho$.

Proof. We shall use the same notations of the above proof, except that $c_{1}$ is replaced by $c_{1}^{\prime}$ and $\mathfrak{B}$ denotes the family of Theorem 2.1, corresponding to the constant $c_{1}^{\prime}$. Our result will follow by analyzing $G$ and $F$ in the above proof. Let $C_{B, t}$ be a general hypersurface section of $B$ by a hypersurface of degree $t$. By Lemma $2.8,(b), g^{-1}$ is defined by a linear subsystem of hypersurface sections of $B$ by hypersurfaces of degree $t$, containing an absolutely irreducible divisor. Let $C_{B, t}^{\prime}$ denote one such divisor. Then $g^{*}\left(C_{B, t}^{\prime}\right) \sim C_{V}+G^{\prime}, G^{\prime}>0$ and every component of $G^{\prime}$ is contracted by $g$. $g^{*}\left(C_{B, t}^{\prime}\right) \sim g^{*}\left(C_{B, t}\right) \sim \operatorname{tg} *\left(C_{B}\right) \sim C_{V}+G$, where $G$ is a positive divisor in the last proof. Hence $G=G^{\prime}$ and every component of $G$ is contracted by $g$. When that is so, every component of $G$ is contracted by $\rho^{\prime} \circ g=\rho$ as $h$ is a birational morphism and $f$ has the property (c) of Proposition 2.1. The divisor $Z$ in this case is an absolutely irreducible divisor in $\Lambda\left(c_{1}^{\prime} Y\right)$ by our assumption. It follows that $\rho^{*}\left(\operatorname{stc}_{1}^{\prime} Y\right) \sim$ $\rho^{*}(s t Z) \sim \rho^{-1}[s t Z]+F$, where every component of $F$ is contracted by $\rho$. It follows that $\rho^{*}\left(s t c_{1}^{\prime} Y\right) \sim C_{V}+F^{\prime}, F^{\prime}>0$, and every component of $F^{\prime}$ is contracted by $\rho$. Since there are only finitely many possibilities for $s$ 
and $t$, we define $c_{3}$ to the L.C.M. of the $s t c_{1}^{\prime}$. Then $c_{3}$ has the desired property.

$\S 4.5$

We shall prove first a Riemann-Roch type inequalities for a semiample divisor on a non-singular projective variety $V$, which coincides with the $\chi$-value in the first two highest degree terms. This unfortunately depends not only on the polynomial $\chi(V, \mathcal{O}(m X))$ but also on a choice of a very ample $V$-divisor. But it provides us with a sharper estimation of $\ell(r Y)$ than that of [13] whenever $(U, Y) \in \Omega$ in view of Theorem 2.1 (cf. also [13], Lemma 5.2). The inequalities will be used to justify the assumption of Theorem 2.2 among others.

Lemma 2.9. Let $V^{n}$ be a non-singular variety and $C$ a very ample $V$ divisor. Then there is a non-negative integer $\bar{s}$, which depends only on $C^{(n)}$ and $I\left(K_{V}, C^{(n-1)}\right)$, such that $K_{V}+m C$ is very ample for $m \geqq \bar{s}$.

Proof. There is a non-negative integer $u \leqq n+1$ such that $\ell\left(K_{r^{*}}+\right.$ $u C)>0$ by the vanishing theorem. Then when we let

$$
s^{\prime}=\left(I\left(K_{V}, C^{(n-1)}\right)+u C^{(n)}\right) \cdot\left(C^{(n)}-2\right)+(u+1) C^{(n)},
$$

$K_{V}+m C$ is very ample on $V$ for $m \geqq s^{\prime}$ (cf. [20], Chap. IX, Corollary, Theorem 13). Our Lemma follows from this at once.

Lemma 2.10. Let $V^{n}$ be a non-singular projective variety, $C$ a very ample $V$-divisor and $X$ a semi-ample divisor on $V$. Let $\bar{s}$ be the integer in Lemma 2.9. Then there is a positive integer $s$, which depends only on $\chi\left(V, \mathcal{O}\left(v_{1} K_{V}+v_{2} X+v_{3} C\right)\right)$, such that $\ell\left(s X-K_{V}-\bar{s} C\right)>0$.

Proof. Let $E$ be an absolutely irreducible non-singular divisor such that $E \sim K_{V}+\bar{s} C$. We have the following well-known equality

$$
\ell(m X)=\ell(m X-E)+\operatorname{dim} \operatorname{Tr}_{E} L(m X) .
$$

Let $X^{\prime}=X . E . \ell(m X-E)=0$ implies that $\ell(m X) \leqq \ell\left(E ; m X^{\prime}\right) . \quad X^{\prime(n-1)}$ and $I\left(K_{E}, X^{\prime(n-2)}\right)$ are determined by the polynomial $\chi\left(V, \mathcal{O}\left(v_{1} K_{V}+v_{2} X+v_{3} C\right)\right)$

5) Contents of this paragraph are not new and are essentially reproduction of a part of the works of S. Cutkosky in the special case of semi-ample divisors. The results make dependence of estimations on invariants clear. The lower bound similar to that of Proposition 2.3 and Theorem 2.3, (b) may be obtained also from the results of [19], [22] when we use the main result of [16]. 
and $\bar{s}$. It follows that there is a positive integer $s^{\prime}$, determined only by the given data in our Lemma, such that

$$
\ell\left(E ; m X^{\prime}\right) \leqq X^{\prime(n-1)} m^{n-1} /(n-1) !+s^{\prime} m^{n-2}
$$

for all positive integers by [13], Theorem 2 and Lemma 5.2. Therefore, the equality $\ell(m X-E)=0$ leads to

$$
X^{(n)} m^{n} / n !-Q(m) \leqq X^{(n-1)} m^{n-1} /(n-1) !+s^{\prime} m^{n-2},
$$

where $Q(x)$ is a polynomial with rational coefficients of degree at most $n-1$, which depends only on $X^{(n)}$ and $I\left(K_{V}, X^{(n-1)}\right)$ (cf. [13]). Our Lemma follows from this easily.

Proposition 2.3. Let $V^{n}$ be a non-singular projective variety, $X$ a semi-ample $V$-divisor and $C$ a very ample $V$-divisor. Let $r$ be a positive integer and assume that $r X+D$ is semi-ample on $V$. Then there is a polynomial $R(x)$ of degree at most $n-2$ with rational coefficients, which depends only on $\chi\left(V, \mathcal{O}\left(v_{1} K_{V}+v_{2} X+v_{3} C+D\right)\right)$ such that

$$
\chi(V, \mathcal{O}(r X+D))-R(r) \leqq \ell(r X+D) .
$$

Proof. Let $Z=r X+D$. By Lemma 2.9, we can find a positive integer $\bar{s}$ depending on the polynomial above such that $\Lambda\left(K_{V}+\bar{s} C\right)$ contains an absolutely irreducible ample non-singular divisor $E$. We have a wellknown equality

$$
\ell(Z+E)=\ell(Z)+\operatorname{dim} \operatorname{Tr}_{E} L(Z+E) .
$$

There is a positive integer $u \leqq n+1$ such that $\ell\left(K_{V}+u X\right)>0$ by the vanishing theorem. Then we have $\ell\left(E ; K_{E}+(u X+Z) . E\right) \geqq \operatorname{dim} \operatorname{Tr}_{E} L(Z+E)$ by our choice of $u$ and by the adjunction formula. It follows that

$$
\ell(Z) \geqq \ell(Z+E)-\ell\left(E ; K_{E}+(u X+Z) . E\right) .
$$

$Z+E \sim K_{V}+Z+\bar{s} C . \quad u X+Z$ and $Z+\bar{s} C$ are both semi-ample on $V$ by our assumption. When that is so, $\ell\left(E ; K_{E}+(u X+Z) . E\right)=\chi\left(E, \mathcal{O}\left(K_{E}+\right.\right.$ $(u X+Z) . E)$ and $\ell(Z+E)=\chi(V, \mathcal{O}(Z+E))$ by the vanishing theorem. Then a straight forward calculations show the existence of $R(x)$ as asserted in our Proposition.

Proposition 2.4. Let the notations and assumptions be as in the above Proposition. Let $s$ be the positive integer of Lemma 2.10. Let $r$ be a positive integer such that $r-s>0$ and assume that $(r-s) X+D$ is semi- 
ample on $V$. Then there is a polynomial $R^{\prime}(x)$ of degree at most $n-2$ with rational coefficients, which depends only on the polynomial $\chi\left(V, \mathcal{O}\left(v_{1} K_{V}+\right.\right.$ $\left.v_{2} X+v_{3} C+D\right)$ ), such that

$$
\ell(r X+D) \leqq \chi(V, \mathcal{O}(r X+D))+R^{\prime}(r) .
$$

Proof. Letting $s X \sim K_{V}+\Delta, \Delta>0$, we shall use the same notations of the proof of the above Proposition. We first observe that $Z+K_{V}+\bar{s} C \sim$ $2 K_{V}+\bar{s} C+Z-s X+\Delta$. Let $F=2 K_{V}+\bar{s} C+Z-s X$. As $L(F)$ is a vector subspace of $L\left(K_{V}+\bar{s} C+Z\right)$ and as $K_{V}+\bar{s} C \sim E$ by definition, we find an inequality

$$
\ell(Z+E) \geqq \ell(Z)+\operatorname{dim} \operatorname{Tr}_{E} L(F)
$$

from the first equality in the above proof. We have an exact sequence of invertible sheaves

$$
0 \longrightarrow \mathcal{O}(F-E) \longrightarrow \mathcal{O}(F) \longrightarrow \mathcal{O}_{E}(F . E) \longrightarrow 0
$$

$Z-s X=(r-s) X+D$ is semi-ample on $V$. Then higher cohomology groups of all three sheaves above vanish by the vanishing theorem as $K_{V}+\bar{s} C$ is very ample. It follows in particular that $\operatorname{dim} \operatorname{Tr}_{E} L(F)=$ $\ell\left(E ; K_{E}+(Z-s X) . E\right)$. Then straight forward calculations show the existence of $R^{\prime}(x)$ as claimed in our Proposition.

TheOREm 2.3. Let $\mathfrak{B}^{*}$ be an irreducible algebraic family consisting of triplet $(V, X, D)$ where $V$ is a non-singular subvariety of a fixed projective space and $X, D$ are $V$-divisors respectively. Then there are two polynomials $R(x)$ and $R^{\prime}(x)$ with rational coefficients of degrees at most $n-2$ with the following properties.

(a) Let $(V, X, D)$ be a member of $\mathfrak{B}^{*}$ such that $X$ is semi-ample on $V$ and $r$ a positive integer. Assume that $r X+D$ is semi-ample on $V$. Then we have

$$
\ell(r X+D) \geqq \chi(V, \mathcal{O}(r X+D))-R(r) .
$$

(b) There is a positive integer $s$ with the following properties. Let $(V, X, D)$ be a member of $\mathfrak{B}^{*}$ such that $X$ is semi-ample on $V$. Let $r$ be a positive integer such that $r-s>0$ and assume that $(r-s) X+D$ is semiample on $V$. Then we have

$$
\ell(r X+D) \leqq \chi(V, \mathcal{O}(r X+D))+R^{\prime}(r) .
$$


Proof. This follows at once from Lemmas 2.9, 2.10 and Propositions 2.3, 2.4 .

CoRollary. There are finitely many pairs $\left(R_{i}(x), R_{i}^{\prime}(x)\right)$ of polynomials with rational coefficients of degrees at most $n-2$ and a positive integer $c_{4}$ with the following properties: Whenever $(U, Y) \in \Omega$, there is an index $i$ such that

$$
\ell(r Y) \geqq d r^{n} / n !-\xi r^{n-1} / 2 .(n-1) !-R_{i}(r)
$$

for all positive integers $r$ and

$$
\ell(r Y) \leqq d r^{n} / n !-\xi r^{n-1} / 2 \cdot(n-1) !+R_{i}^{\prime}(r)
$$

for all positive integers $r$ satisfying $r-c_{4}>0$.

Proof. This follows from the Theorem and from Theorem 2.1.

$\S 5$.

We shall prove here with the aid of the results of Section 4 that there is a positive integer $c_{1}^{\prime}$ and a linear subsystem of $\Lambda\left(c_{1}^{\prime} Y\right)$ without fixed component for every $(U, Y) \in \Omega$ having two properties (a), (b) of Proposition 2.2. As a consequence, the assumption in Theorem 2.2 is fulfilled when we take $c_{1}^{\prime} \geqq c_{1}$.

We start with the following situation. We let $s_{0}=c_{1}$. According to Proposition 2.2, there is a vector subspace $L_{0}^{\prime}$ of $L\left(s_{0} Y\right)$ for every $(U, Y) \in \Omega$, such that a non-degenerate rational map $f_{0}$ of $U$ defined by $L_{0}^{\prime}$ is a birational transformation of $U$ and that the graph of $f_{0}$ has the property (c) of Proposition 2.1.

We shall fix one member $(U, Y)$ in $\Omega$ for the sake of our discussions. Let $\Lambda_{0}$ be the linear subsystem of $A\left(s_{0} Y\right)$ defined by $L_{0}^{\prime}$ and $B$ the fixed part of $\Lambda_{0}$. Let $A_{0}$ be a general fixed divisor of $\Lambda_{0 \text {,red }}$. Let $h$ be a function $U$ such that $\operatorname{div}(h)=s_{0} Y-\left(A_{0}+B\right)$. Then $L_{0}=h . L_{1}^{\prime}$ is a vector subspace of $L\left(A_{0}+B\right)$ containing 1 . Let $\left\{1=g_{0}, g_{1}, \cdots, g_{\omega}\right\}$ be a basis of $L_{0}$. We shall also assume that $B \neq 0$.

LEMma 2.11. Let $B^{\prime}$ be a U-divisor with $0<B^{\prime} \leqq B$. Assume that $A\left(s T+B^{\prime}\right)$ has no fixed component for some $s>0$. Then the vector space $L^{\prime}$ defined by

$$
L^{\prime}=\sum_{0}^{\omega} L\left(s Y+B^{\prime}\right) g_{i}
$$


has the properties (a), (b) of Proposition 2.2. Moreover, $L^{\prime}$ defines a linear subsystem of $\Lambda\left(s Y+A_{0}+B^{\prime}\right)$ without fixed component.

Proof. The first part of our Lemma follows from Lemma 2.5, as $L(s Y)$ and hence $L\left(s Y+B^{\prime}\right)$ defines a birational transformation of $U$ by the definition of $\Omega$. The second assertion follows at once from the definitions.

LeMma 2.12. Let $B=\sum b_{i} B_{i}$ be the reduced expression for $B$. Then the $b_{i}, \sum b_{i}$ and $I\left(B_{i}, Y^{(n-1)}\right)$ are bounded above by $s_{0} Y^{(n)}$.

Proof. This follows from the linear equivalence relation $s_{0} Y \sim A_{0}+B$ and from the fact that $Y$ is an ample Cartier divisor on $U$.

LEMMA 2.13. Let $B_{1}$ be a component of $B$ and $s$ a positive integer such that $s>s_{0}$. Then $A\left(s Y-B_{1}\right)$ is not empty, and when $F-B_{1}$ is the fixed part of $A\left(s Y-B_{1}\right)$, it satisfies $0<F \leqq B$. Moreover, when we denote by $\Lambda^{\prime}$ the minimum sum of $\Lambda\left(\left(s-s_{0}\right) Y\right)$ and $\Lambda_{0, \text { red }}$, we have the following relations:

$$
A(s Y) \supset A(s Y-F)+F \supset A^{\prime}+B .
$$

Proof. $s Y \sim\left(s-s_{0}\right) Y+s_{0} Y$ and $s_{0} Y \sim A_{0}+B$. It follows that $A\left(s Y-B_{1}\right)$ is not empty. $Y$ defines a complete linear system without fixed component and the same is true with $A_{0}$ by our definitions. Therefore, when we note that $s Y \sim\left(s-s_{0}\right) Y+A_{0}+\left(B-B_{1}\right)+B_{1}$, we must have $F-B_{1} \leqq B-B_{1}$. When that is so, $\Lambda(s Y) \supset \Lambda(s Y-F)+F \supset \Lambda^{\prime}+B$.

LEMMA 2.14. Using the same notations and assumptions of the above Lemma, we can find a positive integer rs, depending only on $s$ and $\Omega$, such that

$$
\ell((r s-s) Y+F)>\ell(r s-s) Y) .
$$

Proof. By the definition of $F, \Lambda(s Y-F)$ has no fixed component. Moreover, it contains $\Lambda^{\prime}+(B-F)$ as a linear subsystem by the above Lemma. By the definition of $\Lambda^{\prime}$ and by the fact that $\Lambda_{0 \text {, red }}$ is not composed of a pencil as $L_{0}^{\prime}$ defines a birational transformation, $\Lambda(s Y-F)$ is not composed of a pencil. It follows that a general divisor $E$ of $\Lambda(s Y-E)$ is absolutely irreducible by a theorem of Bertini. Let $m$ be a positive integer such that $m>s$ and assume that $\ell((m-s) Y+F)=\ell((m-s) Y)$. When we note that $E+F \sim s Y$, we find that the following equality holds:

$$
\ell((m-s) Y)+\operatorname{dim} \operatorname{Tr}_{E} L(m Y)=\ell(m Y) .
$$


In order to show that this is impossible beyond a certain value of $m$, we shall seek an upper bound of $\operatorname{dim} \operatorname{Tr}_{E} L(m Y)$.

Let $g: V \rightarrow U$ be a desingularization of $U$ with the following properties: (i) $g$ is a birational morphism; (ii) The complete linear system $\Lambda$ on $V$ consisting of counter images of divisors in $A(s Y-F)$ by $g$ has the property that $A_{\text {red }}$ has no base point (cf. [7]). Then $g^{-1}[E]=Z$, which is a general divisor of $\Lambda_{\text {red }}$, is an absolutely irreducible and non-singular divisor by the theorems of Bertini. When we let $X=g^{*}(Y)$, there is a positive divisor $G$ with

$$
A+G \subset \Lambda(s X), \quad G \geqq g^{-1}[F],
$$

as $E+F \sim s Y$. $A$ and hence $\Lambda(s Y-F)$ defines a birational transformation of $U$. It follows that $A$ defines a birational transformation of $V$. In order to apply Proposition 1.4, we let $m=r s$ where $r$ is a positive integer. According to the Proposition, there is a positive integer $v$, which depends only on $s$ and the polynomial $\chi(V, \mathcal{O}(t X))$ such that

$$
\ell(Z ; r s X . Z) \leqq\left(I\left(Z, X^{(n-1)}\right) s^{n-1} /(n-1) !\right) r^{n-1}+v r^{n-2}
$$

$\chi(V, \mathcal{O}(t X))$ is one of the finitely many polynomials determined by $d$ and $\xi$ (cf. [13], Lemma 5.2). We choose the largest possible $v$ among finitely many possible $v$ 's so that it is determined uniquely by $s, d$ and $\xi$. We have

$$
I\left(E, Y^{(n-1)}\right)=I\left(s Y-F, Y^{(n-1)}\right) \leqq s Y^{(n)}-1,
$$

and $I\left(E, Y^{(n-1)}\right)=I\left(Z, X^{(n-1)}\right)$. Moreover, $\operatorname{dim} \operatorname{Tr}_{E} L(m Y) \leqq \operatorname{dim} \operatorname{Tr}_{Z} L(m X) \leqq$ $\ell(Z ; m X . Z)$. It follows that

$$
\operatorname{dim} \operatorname{Tr}_{E} L(r s Y) \leqq\left(s^{n} d-s^{n-1}\right) r^{n-1} /(n-1) !+v r^{n-2},
$$

where $d=Y^{(n)}$.

Let $m-s=r s-s>c_{4}$. Then by the Corollary to Theorem 2.3, we have

$$
\begin{aligned}
& P(r s-s)+R_{i}^{\prime}(r s-s)+\left(s^{n} d-s^{n-1}\right) r^{n-1} /(n-1) !+v r^{n-2} \\
& \quad \geqq P(r s)-R_{\imath}(r s),
\end{aligned}
$$

where $\left(R_{i}(x), R_{i}^{\prime}(x)\right)$ is one of the finitely many pairs of polynomials of the Corollary. Since these two polynomials are of degree at most $n-2$ with rational coefficients, straight forward calculations show the existence of the smallest positive integer $r_{0}$ such that the above inequality is impossible for $r \geqq r_{0}$, irrespective of the choice of the pair $\left(R_{i}(x), R_{i}^{\prime}(x)\right)$. This proves our Lemma. 
CoRollary 1. With the same notations and assumptions of our Lemma, there is a positive divisor $F^{\prime}$ with $B \geqq F \geqq F^{\prime}>0$ such that $\Lambda((r-s) Y$ $\left.+F^{\prime}\right)$ has no fixed component.

Proof. This follows at once from our Lemma.

Corollary 2.

(a) $\sum_{0}^{\omega} L\left((r s-s) Y+F^{\prime}\right) g_{i}=L_{1}^{\prime}$ has the properties (a), (b) of Proposition 2.2 .

(b) $L_{1}^{\prime}$ is a vector subspace of $L\left((r s-s) Y+A_{0}+B\right)$. When $\Lambda_{1}$ is the linear subsystem of $\Lambda\left(\left(r s-s+s_{0}\right) Y\right)$ defined by $L_{1}^{\prime}$, the fixed part $B_{(1)}$ of $\Lambda_{1}$ is given by $B-F^{\prime}$ and $B>B_{(1)}$.

Proof. (a) follows from Lemma 2.5. The first part of (b) follows from the definition of the $g_{i}$. The latter half of (b) follows from Lemma 2.11 and from the above Corollary 1.

Proposition 2.5. We can find a positive integer $c_{5}$, depending only on $\Omega$, having the following properties:

(a) There is a vector subspace $M$ of $L\left(c_{5} Y\right)$ which has the properties (a), (b) of Proposition 2.2;

(b) $M$ defines a linear subsystem of $\Lambda\left(c_{5} Y\right)$ without fixed component.

Proof. We start by letting $s=s_{0}$ in Lemma 2.11. Let $r s_{0}=s$ be the positive integer of Lemma 2.14. Since $s$ is determined, depending only on $\Omega$, the same is true with $s_{1}$. Let $L_{1}$ be the vector subspace of $L\left(s_{1} Y\right)$, isomorphic to the vector space $L_{1}^{\prime}$ of Corollary 2, Lemma 2.14. The linear subsystem $\Lambda_{1}$ of $\Lambda\left(s_{1} Y\right)$ defined by $L_{1}$ has the fixed part $B_{(1)}$, which satisfies

$$
s_{0} Y^{(n)}>I\left(B, Y^{(n-1)}\right)>I\left(B_{(1)}, Y^{(n-1)}\right)
$$

by Lemma 2.12 and by Corollary 2, Lemma 2.14. Then the above process can be started again with $L_{1}, L\left(s_{1} Y\right)$ and the fixed part $B_{(1)}$ of $\Lambda_{1}$. It can be continued until we get a vector subspace $L_{t}$ of $L\left(s_{t} Y\right)$ such that the linear subsystem $\Lambda_{t}$ of $\Lambda\left(s_{t} Y\right)$ defined by $L_{t}$ has no fixed component. The process terminates at most by $s_{0} Y^{(n)}$-steps, since the degrees of the fixed parts we obtain decrease step by step by (b), Corollary 2, Lemma 2.14. This proves our Proposition.

$\S 6$.

We shall apply here the earlier results of Chapters I and II to the 
subset $\Omega^{\prime}$ which consists of pairs $(U, Y)$ in $\Omega$ such that the singularities of $U$ are only rational singularities.

LEMma 2.15. Let $Z^{n}$ be a normal projective variety, $W$ a non-singular projective variety and $g$ a birational morphism of $W$ on $Z$. Let $Y$ be an ample Cartier divisor on $Z$ and $X=g^{*}(Y)$. Assume that $Z$ has rational singularities only. Then $\chi(W, \mathcal{O}(m X))=\chi(Z, \mathcal{O}(m Y))$ and $h^{i}(W, \mathcal{O}(m Y))=$ $h^{i}(Z, \mathcal{O}(m Y))$ for all $i \geq 0$ and $m$.

Proof. Let $\mathscr{F}=\mathcal{O}_{W} \otimes \mathcal{O}_{W}\left(g^{*}(m Y)\right) \cong \mathcal{O}_{W}(m X) . \quad R^{q} g^{*} \mathscr{F}$ is coherent on $Z$ for $q \geq 0$ and there is a Leray spectral sequence

$$
E_{2}^{p, q}=H^{p}\left(Z, R^{p} g_{*} \mathscr{F}\right) \Longrightarrow E^{p+q}=H^{p+q}(W, \mathscr{F})
$$

(cf. [5]). The well known projection formula shows that

$$
R^{q} g_{*} \mathscr{F} \cong\left(R^{q} g_{*} \mathcal{O}_{W}\right) \otimes \mathcal{O}_{Z}(m Y)
$$

and $R^{q} g_{*} \mathcal{O}_{W}=0$ for $q>0$ by our assumption on singularities of $Z$ (cf. [9]). This implies that $H^{p}\left(Z, R^{q} g_{*} \mathscr{F}\right)=E_{2}^{p, q}=0$ for $q>0$ and the spectral sequence degenerates. It follows that $E_{2}^{p, 0} \cong E^{p}$ for $p \geqslant 0$, which is equivalent to

$$
H^{p}\left(Z, R^{0} g_{*} \mathscr{F}\right) \cong H^{p}(W, \mathscr{F}) \quad \text { for } p \geqslant 0 .
$$

Since $g$ is a birational morphism on a normal projective variety $Z$, $R^{0} g_{*} \mathcal{O}_{W}=g_{*} \mathcal{O}_{W} \cong \mathcal{O}_{Z}$ and $R^{0} g_{*} \mathscr{F} \cong \mathcal{O}_{Z}(m Y)$ by the above projection formula. Our Lemma follows from this and from the definition of the Euler-Poincare characteristic.

Let $\Omega$ be the set of pairs $(U, Y)$ defined in Chap. II, Section 2 and $\Omega^{\prime}$ a subset of $\Omega$. Let $\mathfrak{B}$ be a finite union of irreducible algebraic families with the following properties:

(a) Members of $\mathfrak{B}$ are of the form $(V, X)$ where $V$ is a non-singular subvariety of a fixed projective space and $X$ a $V$-divisor;

(b) Whenever $(U, Y) \in \Omega^{\prime}$, there is a member $(V, X) \in \mathfrak{B}$ and a birational morphism $\rho: V \rightarrow U$ such that $X \sim \rho^{*}(Y)$;

(c) There is a positive integer $c$ such that whenever $(U, Y),(V, X)$ and $\rho$ are as in (b), $c X \sim C+G$, where $C$ is a very ample $V$-divisor and $G$ a positive $V$-divisor such that every component of it is contracted by $\rho$;

(d) When $\mathfrak{B}_{i}$ is a component family of $\mathfrak{B}$, it contains a pair $(V, X)$ which corresponds to a member $(U, Y) \in \Omega^{\prime}$ in the sense of (b).

When $V$ satisfies these conditions, we shall say that $\mathfrak{B}$ is an algebraic 
family associated with $\Omega^{\prime}$. When $(U, Y)$ and $(V, X)$ are as in $(\mathrm{b})$, we shall say that $(U, Y)$ corresponds to $(V, X)$ and that $(V, X)$ corresponds to $(U, Y)$. Theorem 2.2 and Proposition 2.5 show that there is an algebraic family $\mathfrak{B}$ which is associated with $\Omega$. When that is so, we can find an algebraic family associated with $\Omega^{\prime}$ at once from $\mathfrak{B}$ by deleting some component families, if necessary. From now on, we shall assume that $\Omega^{\prime}$ is a set of $(U, Y)$ in $\Omega$ such that singularities of $U$ are rational singularities and that $\mathfrak{B}$ is an algebraic family associated with $\Omega^{\prime}$.

Lemma 2.16. Let $\mathfrak{B}_{i}$ be a component family of $\mathfrak{B}$. Let $\left(V_{i}^{\prime}, X_{i}^{\prime}\right)$ be a fixed member of $\mathfrak{B}_{i}$ corresponding to a member $\left(U_{i}^{\prime}, Y_{i}^{\prime}\right)$ in $\Omega^{\prime}$ for each $i$. Then we can find a positive integer $c_{6}$, depending only on $\Omega^{\prime}$, having the following properties:

(i ) $h^{j}\left(V_{i}^{\prime}, \mathcal{O}\left(c_{6} X_{i}^{\prime}\right)\right)=0$ for $j>0$ for all $i$;

(ii) $\Lambda\left(c_{6} X_{i}^{\prime}\right)$ has no base point for all $i$;

(iii) For each $i$, we can find a non-empty open irreducible subfamily $\mathfrak{B}_{i}^{\prime}$ of $\mathfrak{B}_{i}$, such that whenever $(V, X) \in \mathfrak{B}_{i}^{\prime}, \Lambda\left(c_{6} X\right)$ has no base point and $\ell\left(c_{6} X\right)=\ell\left(c_{6} X_{i}^{\prime}\right)$.

Proof. Since $Y_{i}^{\prime}$ is an ample Cartier divisor on a normal projective variety $U_{i}^{\prime}$, (i) and (ii) follow at once from Lemma 2.15. Let $k$ be a field over which every $\mathfrak{B}_{i}$ is defined. Let us omit the index $i$ for the sake of simplicity from this point on. Let $(V, X)$ be a generic pair of $\mathfrak{B}$ over $k$. We have $h^{j}\left(V, \mathcal{C}\left(c_{6} X\right)\right)=0$ for $j>0$ by the upper semi-continuity. It follows that

$$
\ell\left(c_{6} X\right)=\chi\left(V, \mathcal{O}\left(c_{6} X\right)\right)=\ell\left(c_{6} X^{\prime}\right)
$$

by the invariance of the Euler-Poincare characteristic by specialization.

Let $T$ be the support of the Chow-variety of $\Lambda\left(c_{6} X\right)$ and $\left(V^{\prime}, X^{\prime}, T^{\prime}\right)$ a specialization of $(V, X, T)$ over the specialization $(V, X) \rightarrow\left(V^{\prime}, X^{\prime}\right)$ ref. $k$. The invariance of linear equivalence by specialization (cf. [18]) shows that $T^{\prime}$ is contained in the support of the Chow-variety of $\Lambda\left(c_{6} X^{\prime}\right)$. Then $\ell\left(c_{6} X\right)=\ell\left(c_{6} X^{\prime}\right)$ implies that $T^{\prime}$ is the support of the Chow-variety of $A\left(c_{6} X^{\prime}\right)$. The absence of base points in $\Lambda\left(c_{6} X\right)$ follows from this easily. The rest of (iii) then follows from the Lemma in Appendix 1.

THEOREM 2.4. We can find a positive integer $c_{7}$ such that whenever $(U, Y)$ is a member of $\Omega^{\prime}, c_{7} Y$ is very ample on $U$ and $h^{j}\left(U, \mathcal{O}\left(m c_{7} Y\right)\right)=0$ for $j>0$, whenever $m$ is a positive integer. 
Proof. By applying the above Lemma finitely many times, we can find a positive integer $c^{\prime}$ such that whenever $(V, X)$ is a member of $\mathfrak{B}$, corresponding to a member $(U, Y)$ of $\Omega^{\prime}$, then $\Lambda\left(c^{\prime} X\right)$ has no base point. Let $\rho$ be the birational morphism of $V$ on $U$. $\Lambda\left(c^{\prime} X\right)$ consists of $\rho^{*}(Z)$ with $Z \in\left(c^{\prime} Y\right)$. Hence $\Lambda\left(c^{\prime} Y\right)$ cannot have a base point.

When we use the Riemann-Roch inequality $\left|\ell(r Y)-d r^{n}\right| n ! \mid \leq \phi(r)$ in Section 2, we can find a positive integer $c^{\prime \prime}$ such that

$$
H^{0}\left(U, \mathcal{O}\left(m c^{\prime} Y\right)\right) \otimes H^{0}\left(U, \mathcal{O}\left(c^{\prime} Y\right)\right) \longrightarrow H^{0}\left(U, \mathcal{O}\left((m+1) c^{\prime} Y\right)\right)
$$

is surjective and $h^{1}\left(U, \mathcal{O}\left(m c^{\prime} Y\right)\right)=0$ for $i>0, m \geq c^{\prime \prime}$ by [10], Chap. II, Proposition 1 and Theorem 1. The first implies in particular that $m c^{\prime} Y$ is very ample for such $m$ as $U$ is normal. Our theorem follows from this.

\section{ApPendix I}

Lemma. Let $[M ;(W, Z), P]$ be an irreducible algebraic family satisfying the following conditions: (i) For every $u^{\prime}$ in $M, W\left(u^{\prime}\right)$ is absolutely irreducible and non-singular in codimension 1 ; (ii) $Z\left(u^{\prime}\right)$ is a positive divisor on $W\left(u^{\prime}\right)$. Then there is a non-empty open subset $M^{\prime}$ of $M$ and irreducible algebraic families $\left[M^{\prime} ; T, P \times P^{\prime}\right]$ and $\left[M^{\prime} ; F, P\right]$ with the following properties:

(a) Whenever $u^{\prime}, u^{\prime \prime}$ are points of $M^{\prime}, \ell\left(Z\left(u^{\prime}\right)\right)=\ell\left(Z\left(u^{\prime \prime}\right)\right)$ and $T\left(u^{\prime}\right)$ is a non-degenerate rational map of $W\left(u^{\prime}\right)$ into a projective space $P^{\prime}$ defined by $L\left(Z\left(u^{\prime}\right)\right)$.

(b) When $u^{\prime}$ is a point of $M^{\prime}, F\left(u^{\prime}\right)$ is the fixed part of $\Lambda\left(Z\left(u^{\prime}\right)\right)$.

Proof. The first part of (a) follows from Proposition 1, Appendix in [16], when we take a suitable open subset $M_{0}$ of $M$.

Let $k$ be an algebraically closed field of definition for $[M ;(W, Z), P]$ over which $M_{0}$ is $k$-open. Let $u$ be a generic point of $M$ over $k$. As $W(u)$ is non-singular in codimension $1, L(Z(u))$ has a basis over $k(u)$. It follows that there is a non-degenerate rational map $f_{u}$ of $W(u)$, defined over $k(u)$, mapping $W(u)$ into a projective space $P^{\prime}$. Let $T_{u}$ be the graph of $f_{u}$. Since $T_{u}$ is defined over $k(u)$, there is an absolutely irreducible subvariety $T$ of $M \times P \times P^{\prime}$, defined over $k$, such that $T(u)=T_{u}$. The set $M_{1}$ of points $u^{\prime}$ of $M$ such that every component of $u^{\prime} \times P \times P^{\prime} \cap T$ is proper and $T\left(u^{\prime}\right)$ is absolutely irreducible is $k$-open on $M$ (cf. Lemmas 
1, 2, Appendix, [16]). When we use the technique of Chow-forms and incidence correspondence (cf. [3]), we see that the set $M_{2}$ of points of $M_{1}$ such that the projection of $T\left(u^{\prime}\right)$ in $P^{\prime}$ is not contained in a hyperplane is $k$-open on $M_{1}$ and hence on $M$. Let $M^{\prime}=M_{0} \cap M_{2}$. For every $u^{\prime}$ in $M^{\prime}, T\left(u^{\prime}\right)$ is the graph of a rational map of $W\left(u^{\prime}\right)$ into $P^{\prime}$ by the compatiblility of specializations with the operation of algebraic projection (cf. [18]). Further, note that when $H^{\prime}$ is a general hyperplane in $P^{\prime}$ with respect to $W\left(u^{\prime}\right), \operatorname{pr}_{W\left(u^{\prime}\right)}\left[T(u) .\left(W\left(u^{\prime}\right) \times H^{\prime}\right)\right]$ is a general member of the linear system on $W\left(u^{\prime}\right)$ which defines $T\left(u^{\prime}\right)$. This shows that the degree of such a general divisor remains constant on $M^{\prime}$ as specializations do not change degrees.

Let $F_{u}$ be the fixed part of $\Lambda(Z(u))$, which is rational over $k(u)$. There is a positive cycle $F$ rational over $k$ on $M \times P$ such that $F(u)=F_{u}$. Let $\left(u^{\prime}, F^{\prime}\right), u^{\prime} \in M^{\prime}$, be a specialization of $(u, F(u))$ over $k$. We can find a generic hyperplane $H$ (resp. $H^{\prime}$ ) in $P^{\prime}$ over $k(u)$ (resp. $k\left(u^{\prime}\right)$ ) such that $\left(u^{\prime}, F^{\prime}, H^{\prime}\right)$ is a specialization of $(u, F(u), H)$ over $k$. When that is so, the compatibility of specializations with the intersection product and algebraic projection, with the additional fact that linear equivalence is preserved by specialization, imply that

$$
\begin{aligned}
\operatorname{pr}_{W(u)} & {[T(u) .(W(u) \times H)]+F(u) } \\
\quad \longrightarrow \operatorname{pr}_{W\left(u^{\prime}\right)} & {\left[T\left(u^{\prime}\right) .\left(W\left(u^{\prime}\right) \times H^{\prime}\right)\right]+F^{\prime} \text { ref. } k, }
\end{aligned}
$$

and the latter is a member of $\Lambda\left(Z\left(u^{\prime}\right)\right)$. When that is so, $F^{\prime}$ is easily seen to be the fixed part of $\Lambda\left(Z\left(u^{\prime}\right)\right)$ and that it is the unique specialization of $F(u)$ over the specialization $u \rightarrow u^{\prime}$ ref. $k$. This shows that every component of $u^{\prime} \times P \cap E$ is proper and $F(u)=F^{\prime}$ by the compatibility of specialization with the operation of intersection product. Thus (b) and the second half of (a) are proved.

\section{APPENDIX II}

Here we shall generalize the contents of Section 2 and Section 3 of [13]. First we shall fix the notations and assumptions. We shall denote by $V^{n}$ a nonsingular projective variety and the $A_{i}(1 \leqq i \leqq n-2)$ semiample $V$-divisors. Let $k$ be an algebraically closed field of definition for $V$ over which the $A_{i}$ are rational. We fix a large positive integer $t$ such 
that $\Lambda\left(t A_{i}\right)$ has no base point and that $L\left(t A_{i}\right)$ defines a birational morphism of $V$ for all $i$. Let the $A_{i}(t)$ be independent generic divisors of the $n-2$ complete linear systems over $k, U^{2}=A_{1}(t) \cdots A_{a-2}(t)$ and $k^{\prime}$ the smallest field which contains $k$ over which $U^{2}$ is defined.

We define $X$ to be a linear combination $\sum_{1}^{n-2} a_{i} A_{i}$ with non-negative coefficient such that at least one of the coefficients is positive. Furthermore, we shall assume that the divisor $X$ satisfies the following two conditions:

$$
I\left(D, X^{(n-1)}\right)=0 \quad \text { implies } \quad I(D, X, U)=0 \quad \text { and } \quad I\left(D, A_{i}, U\right)=0
$$

for all $i$, whenever $D$ is a subvariety of $V$ of codimension 1 .

Remark. We have $\ell\left(K_{V}+u X\right)>0$ for some integer $u$ with $1 \leqq u \leqq$ $n+1$ by the vanishing theorem.

With these essentially all results contained in Sections 2, 3 in [13] are valid as they are. In particular we have the following result:

Proposition. Let $u$ be as in the above Remark and $\sum_{i} D_{i}$ an element of $\Lambda\left(K_{V}+u X\right)$. Then there is a positive $V$-divisor $\sum_{j} D_{j}$ such that $\sum_{i} D_{i}-$ $\sum_{j} D_{j}>0$ and the $K_{V}+u X-\sum_{j} D_{j}$ induces on $U$ a numerically effective divisor. The $D_{j}$ satisfy $I\left(D_{j}, X^{(n-1)}\right)=0$ and hence

$$
I\left(D_{j}, X, U\right)=I\left(D_{j}, A_{i}, U\right)=0 \quad \text { for all } i .
$$

Very little change is needed in the original discussions. We shall list below some remarks or changes needed.

$\S 2$ Lemmas 2.1, 2.2, 2.3, 2.4 and (a) of Lemma 2.5 are valid as they are, together with the Corollaries of Lemma 2.1, except the following deletion and change are made:

In Corollary 2, Lemma 2.1, the conclusion should be $I\left(K_{V}+n X+\right.$ $D, D, U) \geqq 0$.

In Lemma 2.2, (i), delete " $D$ ' is an absolutely irreducible curve."

In Lemma 2.4 , (c) delete " $C=D_{i} . U$ and."

Lemma 2.5, (b) and (c) have to be slightly modified as follows:

Revised version of Lemma 2.5, (b) and (c):

(b) $I\left(D_{i}, X^{(n-1)}\right)=0$ and every component $C$ of $D_{i}$.U satisfies $I(C, X)=0$.

(c) Each component of $D_{i} . U$ is an exceptional curve of the first kind on $U$.

Proof. We shall omit the index $i$. (a) implies that $I\left(K_{V}+u X, D, U\right)<0$. 
If $I\left(D, X^{(n-1)}\right)>0$, we have $I\left(K_{V}+u X, D, U\right) \geqq 0$ by Lemma 2.4 , (a) and (c). It follows that $I\left(D, X^{(n-1)}\right)=0$. This implies that $I(D, X, U)=0$ by our assumption on $X$ and $I(C, X)=0$ follows from Lemma 2.4, (a).

$C^{(2)}<0$ follows from the same reason as in the original proof. Then $I\left(K_{V}, C\right)<0$ by the assumption of the Lemma by (b). The divisor class of $K_{V}+\sum_{1}^{n-2} A_{i}(t)$ induces that of $K_{U}$ on $U$. (b) and the assumption on $X$ implies that $I\left(D, A_{j}, U\right)=0$ for all $j$. When that is so, we have $I\left(K_{E}, C\right)<0$. The rest of the original proof of (c) then works as they are.

$\S 3$ Virtually no change is required except that in the proofs of Lemmas 3.2 and $3.3, K_{U} \sim\left(K_{V}+t \sum_{1}^{n-2} A_{i}\right) . U$ and hence $K^{\prime \prime}+t \sum_{1}^{n-2} A_{i}^{\prime \prime} \sim$ $K_{U^{\prime \prime}}$ when we follow notations and conventions there. Our Proposition is thus a generalized version of Corollary 1 to Proposition 3.2.

\section{REFERENCES}

[1] M. Artin, Some numerical criteria for contractability of curves on algebraic surfaces, Amer. J. Math., 84 (1962), 485-496.

[2] — Isolated rational singularities of surfaces, Amer. J. Math., 88 (1966), 129136.

[ 3 ] W. L. Chow and v. d. Waerden, Zur Algebraischen Geometrie IX, Math. Ann., 113 (1937), 692-704.

[4] R. Elkik, Singularités rationelles et deformations, Invent. Math., 47 (1976), 139147.

[ 5 ] A. Grothendieck and J. Dieudonne, Éléments de géometrie algébrique, Publ. Math. IHES, Paris.

[6] H. Grauert and O. Riemenschneider, Verschwindungssätze für analytische Kohomologiegruppen auf Komplexen Raumen, Invent. Math., 11 (1970), 262-292.

[ 7] H. Hironaka, Resolution of singularities of an algebraic variety over a field of characteristic zero, I-II, Ann. of Math., 79 (1964), 109-326.

[ 8 ] Y. Kawamata, A generalization of Kodaira-Ramanujam's vanishing theorem, Math. Ann., 261 (1982), 43-46.

[ 9 ] G. Kempf, Toroidal embeddings, I, Springer Lecture Notes, 339 (1973), 41-52.

[10] S. Kleiman, Toward a numerical theory of ampleness, Ann. of Math., 84 (1966), 293-344.

[11] K. Kodaira, On a differential geometric method in the theory of analytic stacks, Proc. Nat. Acad. Sci., U.S.A., 39 (1953), 1268-1273.

[12] J. Kollár, Toward moduli of singular varieties, Thesis at Brandeis, 1983, to appear soon.

[13] — and T. Matsusaka, Riemann-Roch type inequalities, Amer. J. Math., 105 (1983), 229-252.

[14] D. Lieberman and D. Mumford, Matsusaka's big theorem, Proc. Symp. Pure Math., 29 (1975), 513-530.

[15] T. Matsusaka and D. Mumford, Two fundamental theorems on deformations of polarized varieties, Amer. J. Math., 86 (1964), 668-684.

[16] T. Matsusaka, Polarized varieties with a given Hilbert polynomial, Amer. J. Math., 94 (1972), 1027-1076. 
[17] M. Rosenlicht, Equivalence relations on algebraic curves, Ann. of Math., 56 (1952), 169-191.

[18] G. Shimura, Reduction of algebraic varieties with respect to a discrete valuation of the basic field, Amer. J. Math., 77 (1955), 134-176.

[19] E. Viehweg, Vanishing theorems, J. Reine Angew. Math., 335 (1982), 1-8.

[20] A. Weil, Foundations of Algebraic Geometry, Amer. Math. Soc. Colloquium Publ., $29(1960)$.

[21] O. Zariski, Foundations of a general theory of birational correspondences, Trans. Amer. Math. Soc., 53 (1943), 490-542.

[22] T. Fujita, Semipositive line bundles, J. Fac. Sci., Univ. Tokyo, 30 (1983), 353-378.

[23] T. Matsusaka, A note and a correction to Riemann-Roch type inequalities, Amer. J. Math., 106 (1984), 1265-1268.

[24] A. Seidenberg, The hyperplane sections of normal varieties, Trans. Amer. Math. Soc., 69 (1950), 357-386.

Department of Mathematics

Brandeis University

Waltham, Massachusetts 02154

U.S.A. 